\title{
Renormalization group invariants and sum rules in the deflected mirage mediation supersymmetry breaking
}

\author{
Katri Huitu, $1 *$ P. N. Pandita, ${ }^{2} \dagger$ and Paavo Tiitola $1 \ddagger$ \\ 1 Department of Physics, and Helsinki Institute of Physics, \\ P. O. Box 64, FIN-00014 University of Helsinki, Finland and \\ 2 Centre of High Energy Physics, Indian Institute of Science, Bangalore 560 012, India
}

\begin{abstract}
We examine the deflected mirage mediation supersymmetry breaking (DMMSB) scenario, which combines three supersymmetry breaking scenarios, namely anomaly mediation, gravity mediation and gauge mediation using the one-loop renormalization group invariants (RGIs). We examine the effects on the RGIs at the threshold where the gauge messengers emerge, and derive the supersymmetry breaking parameters in terms of the RGIs. We further discuss whether supersymmetry breaking mediation mechanism can be determined using a limited set of invariants, and derive sum rules valid for DMMSB below the gauge messenger scale. In addition we examine the implications of the measured Higgs mass for the DMMSB spectrum.
\end{abstract}

PACS numbers: 11.30.Pb, 12.60.Jv, 14.80.Ly

\section{INTRODUCTION}

Supersymmetry remains at present a favored solution to the hierarchy problem of the standard model (SM). Supersymmetry is obviously a broken symmetry, as none of the superpartners of the SM particles have been experimentally observed so far. The idea of weak scale supersymmetry, as embodied, for example, in the minimal supersymmetric standard model (MSSM), as a solution to the gauge hierarchy problem has already been partly tested at the Large Hadron Collider (LHC) [1, 2. The most minimal constrained model is found to be disfavoured, and the emphasis in the studies has moved to other well motivated models.

The mechanism of supersymmetry breaking, which is crucial for determining the masses of the superpartners of the SM particles, is not known at present. There are different viable models of supersymmetry breaking in which supersymmetry breaking is mediated by a specific interaction in a hidden sector and is communicated to the visible sector via some mediator fields. Well known examples of hidden sector supersymmetry breaking include gravity mediated supersymmetry breaking [3, gauge mediated supersymmetry breaking [4, and anomaly mediated supersymmetry breaking [5, respectively. It is usually assumed that one mediation mechanism dominates, which depends on the type of problem that one wishes to address in the context of MSSM. In fact, it may well be that a single mediation mechanism does not dominate. In some situations the anomaly mediated and gravity mediated contributions to the supersymmetry breaking can coexist and manifest at a comparable value, leading to what is known as mirage mediation, a situation that naturally arises in some string theories [6. The name derives from the fact that soft gaugino masses unify at a scale (the mirage scale) which is lower than the grand unification or GUT scale. Models based on mirage mediation can solve the tachyonic slepton mass problem of the anomaly mediation models. The mirage mediation models have been studied extensively in the literature $7-10$.

However, in the absence of any definite experimental indication regarding the mass spectrum of the sparticles, it is important to consider the case where all the three types of supersymmetry breakings contribute to the soft masses, and ultimately determine the mass spectrum of the supersymmetric partners of the SM states. Such a scenario has been dubbed as deflected mirage mediation [11. In the deflected mirage mediation the gaugino mass unification is deflected by the threshold effect from the messenger fields associated with the gauge mediation contribution. The messenger fields are included at scales $\mu>\mu_{\text {mess }}$, and the renormalization group running is affected by the threshold corrections at this scale. It is worthwhile to note that in a broad class of supergravity models, which might be realized in string theory, the contributions to soft supersymmetry breaking masses from the three different mediation mechanisms are comparable [12. This makes the study of deflected mirage mediation models rather compelling.

\footnotetext{
* Electronic address: katri.huitu@helsinki.fi

$\dagger$ Electronic address: pandita@iucaa.ernet.in

\#lectronic address: paavo.tiitola@helsinki.fi
} 
As discussed above, in order to predict the masses of the superpartners of the SM particles, it is essential to understand the nature of supersymmetry breaking. The usual approach to this has been to consider a particular model of supersymmetry breaking, e.g. the gravity mediated supersymmetry breaking model (usualy referred to as mSUGRA) with a limited number of parameters at the high scale (possibly the GUT scale), and evolve these, using renormalization group equations, to the electroweak scale and fit them to the data [13 19]. This method, the so called "top-down" approach has some drawbacks, namely that the reliability of the fit decreases with the increase of the number of parameters at the high scale. This approach is also sensitive to uncertainities of the measured quantities, which includes gauge couplings at the electroweak scale and the masses of the SM particles.

An alternative approach, which is complementary to the "top-down" approach, that has been advocated involves the measured masses at the elctroweak scale and evolve these to the high scale where supersymmetry is broken [20 23. The resulting structure is then analyzed and conclusions about the underlying theory at high scales obtained. This approach, which can be called "bottom-up" approach, has uncertainities resulting from the present experimental uncertainities in the measurement of gauge and Yukawa couplings.

Another alternative to obtain information on the nature of supersymmetry breaking is to obtain specific relations among the masses of the superpartners of the SM particles which result from the structure of the underlying theory at the high scale. In this approach the Yukawa couplings of the first- and second-generations are usually ignored and the renormalization group is used to evolve the parameters from high scale to the low scale, and then specific relations among sparticle masses are derived based on the particular theory at the high scale 24 31. In this context it has also been pointed out that there are a set of combinations of parameters of a supersymmetric model that are renormalization group invariant (RGI) at the 1-loop level [32 38]. Although the argument regarding RGI holds only at the leading-log order, 2-loop corrections are expected to be small and are likely to be negligible compared with the experimental uncertainities. Thus, these higher order effects can either be neglected or absorbed into a shift of the measured values of the renormalization group invariants. It has been argued that these RGIs can be used to extract the parameters of different supersymmetry breaking models at the high scale, and thereby establish the mechanism of supersymmetry breaking. RGIs can also be used for constructing sum rules relating the particle masses, and they are discussed in this context in 25, 26, 28, 37, 39.

In this paper we derive the renormalization group invariants for deflected mirage mediation and examine how the appearance of messenger particles at the specific energy scale lower than the GUT-scale affects the invariants. We investigate the GUT-scale parameters of the deflected mirage mediation in terms of the RGIs, and examine validity of sum rules derived previously of the RGIs and derive new ones specific to the deflected mirage mediation. In particular, we derive soft supersymmetry breaking parameters in terms of the RGIs. We also examine the Higgs mass in the deflected mirage mediation scenario to constrain the parameters of the model.

The plan of this paper is as follows. In Section II] we describe the deflected mirage mediation mechanism that we consider in this paper. Here we analyze the soft supersymmetry breaking gaugino and scalar masses of the model, and the boundary conditions on these parameters. Phenomenological implications, which include the Higgs mass, are studied in this Section. In Section III we describe the renormalization group invariants and calculate these invariants for the deflected mirage mediation supersymmetry breaking model. We then use these invariants to solve for the parameters of the model. In Section IV we obtain sum rules for the deflected mirage mediation scenario and discuss some special cases. In Section $\mathrm{V}$ we proceed to compare the predictions of the pure mirage mediation scenario with those of the gravity, AMSB, and the gauge mediation of supersymmetry breaking models, respectively. Finally, in Section VI we present our conclusions.

\section{DEFLECTED MIRAGE MEDIATION}

The deflected mirage mediation mechanism for supersymmetry breaking mechanism involves contributions of comparable scale from gravity mediation, anomaly mediation and gauge mediation as opposed to the mirage mediation which excludes the contribution from gauge mediation The quantity

$$
\alpha_{m}=m_{3 / 2} /\left(M_{0} \log M_{P} / m_{3 / 2}\right),
$$

parametrises the anomaly to gravity mediation ratio, while $M_{0}$ describes the mass scale of soft supersymmetry breaking terms [40. Here $m_{3 / 2}$ is the gravitino mass and $M_{P}$ the reduced Planck mass. The ratio of the gauge mediated contribution to its anomaly mediated counterpart is parametrised by $\alpha_{g}$. It is related to the messenger fields by the equation

$$
\left|\alpha_{g}\right|=\Lambda / m_{3 / 2}
$$


where $\Lambda$ is a mass scale associated with the messenger fields. The absolute value is allows $\alpha_{g}$ to have negative values. The parameters $\alpha_{m}$ and $\alpha_{g}$ can be considered continuous but in string motivated scenarios they usually have discrete values of the order one [12. The messenger sector is assumed to come in complete GUT representations in order to preserve gauge coupling unification. $N$ represents the number of copies of $\mathbf{5}, \overline{\mathbf{5}}$ representations under $S U(5)$. The original Kaluza-Klein compactification is obtained with $\alpha_{m}=1$ and $N=0$. Both positive and negative values are possible for $\alpha_{g}$. Phenomenological implications of various values of the parameters are discussed in e.g. [12] and [41, especially regarding to the Higgs mass.

Above the messenger scale the renormalization group equations are modified from the MSSM form by adding the number of messenger pairs to the $\beta$-function coefficients $b_{a}$ [40]. Thus,

$$
b_{a}^{\prime}=b_{a}+N,
$$

where $\left\{b_{1}, b_{2}, b_{3}\right\}=\{33 / 5,1,-3\}$. At the GUT scale $\mu_{\mathrm{GUT}}$, the gaugino mass boundary conditions can be written as [1]:

$$
\begin{aligned}
M_{a}\left(\mu_{\mathrm{GUT}}\right) & =M_{0}\left(1+g_{a} \ln \left(M_{P} / m_{3 / 2}\right) b_{a} g_{a} \alpha_{m}\right) \\
& =M_{0}+g_{a}^{2}\left(\mu_{\mathrm{GUT}}\right) \frac{b_{a}^{\prime}}{16 \pi^{2}} m_{3 / 2}, \quad(a=1,2,3) .
\end{aligned}
$$

Here $\mu_{G U T}$ is the high scale which we take to be the GUT scale. Similarly the scalar masses can be written as

$$
\begin{aligned}
m_{i}^{2}\left(\mu_{G U T}\right) & =M_{0}^{2}\left[\left(1-n_{i}\right)-\frac{\theta_{i}}{16 \pi^{2}} \alpha_{m} \ln \left(M_{P} / m_{3 / 2}\right)-\frac{\dot{\gamma}_{i}^{\prime}}{\left(16 \pi^{2}\right)^{2}}\left(\alpha_{m} \ln \left(M_{P} / m_{3 / 2}\right)\right)^{2}\right] \\
& =M_{0}^{2}\left(1-n_{i}\right)-\frac{\theta_{i}}{16 \pi^{2}} m_{3 / 2} M_{0}-\frac{\dot{\gamma}_{i}^{\prime}}{\left(16 \pi^{2}\right)^{2}} m_{3 / 2}^{2}
\end{aligned}
$$

where $n_{i}$ are the modular weights for the scalar masses, $\gamma_{i}$ are the anomalous dimensions,

$$
\gamma_{i}=2 \sum_{a} g_{a}^{2} c_{a}\left(\Phi_{i}\right)-\frac{1}{2} \sum_{l m}\left|y_{i l m}\right|^{2}
$$

in which $c_{a}$ is the quadratic Casimir operator for the field $\Psi_{i}$, and $y_{i l m}$ are the normalized Yukawa couplings. $\dot{\gamma}$, and $\theta_{i}$ are defined as

$$
\begin{aligned}
& \dot{\gamma}_{i}=2 \sum_{a} g_{a}^{4} b_{a} c_{a}\left(\Phi_{i}\right)-\sum_{l m}\left|y_{i l m}\right|^{2} b_{y_{i l m}}, \\
& \theta_{i}=4 \sum_{a} g_{a}^{2} c_{a}\left(\Psi_{i}\right)-\sum_{i, j, k}\left|y_{i j k}\right|^{2}\left(p-n_{i}-n_{j}-n_{k}\right),
\end{aligned}
$$

in which $b_{y_{i l m}}$ is the beta function for the Yukawa coupling $y_{i l m} . \dot{\gamma}_{i}^{\prime}$ is obtained by replacing $b_{a}$ with $b_{a}^{\prime}=b_{a}+N$. For explicit values of $\theta_{i}^{\prime}, \dot{\gamma}_{i}^{\prime}$ see [40]. One-loop renormalization group equations give the boundary condition at the messenger scale $\mu_{\text {mess }}$ for the soft gaugino mass parameters:

$$
M_{a}=g_{a}^{2} \frac{b_{a}^{\prime}}{16 \pi^{2}} m_{3 / 2}+M_{0}\left[1-g_{a}^{2} \frac{b_{a}^{\prime}}{8 \pi^{2}} \log \left(\frac{\mu_{\mathrm{GUT}}}{\mu_{\mathrm{mess}}}\right)\right]+\Delta M_{a} \quad(a=1,2,3)
$$

where

$$
\Delta M_{a}=-N M_{0} \frac{g_{a}^{2}}{16 \pi^{2}} \alpha_{m}\left(1+\alpha_{g}\right) \ln \frac{M_{P}}{m_{3 / 2}}
$$

is a threshold contribution that arises when the messenger fields are integrated out. Similarly, scalar masses receive a threshold correction,

$$
\Delta m_{i}^{2^{j}}=M_{0}^{2} \sum_{a} 2 c_{a}\left(\Psi_{i}\right) N \frac{g_{a}^{4}\left(\mu_{\mathrm{mess}}\right)}{\left(16 \pi^{2}\right)^{2}}\left[\alpha_{m}\left(1+\alpha_{g}\right) \ln \frac{M_{P}}{m_{3 / 2}}\right]^{2} \delta_{i}^{j} .
$$

The gaugino masses unify at the mirage scale 40]

$$
\mu_{\text {mirage }}=\mu_{G U T}\left(\frac{m_{3 / 2}}{M_{P}}\right)^{\alpha_{\mathrm{m}} \rho / 2},
$$


in which

$$
\rho=\frac{1+\frac{2 N g_{0}^{2}}{16 \pi^{2}} \ln \frac{M_{\mathrm{GUT}}}{\mu_{\mathrm{mess}}}}{1-\frac{\alpha_{\mathrm{m}} \alpha_{\mathrm{g}} N g_{0}^{2}}{16 \pi^{2}} \ln \frac{M_{P}}{m_{3 / 2}}} .
$$

When $\rho=1$, this reduces to the mirage scale of pure mirage mediation as the deflection is removed. We note that even if gauge mediation is turned off by setting $\alpha_{g}=0$, mirage mediation is not recovered. This is achieved only by removing the messenger fields by setting $N=0$. This is due to the messenger particles affecting the beta functions and thus anomaly mediation at high scales.

To examine the sparticle spectrum of the deflected mirage mediation we have calculated the soft scalar and gaugino masses using one-loop renormalization group equations and the lightest Higgs mass using the one-loop radiative corrections presented in [42. In Fig. 1] we have plotted the lightest Higgs mass for several values for the parameters $\alpha_{m}, M_{0}$ and $N$ as a function of $\alpha_{g}$. The current experimental limits for the Higgs mass are represented as horizontal dashed lines 43 . Although not all the parameter combinations satisfy the experimental bounds on the Higgs mass, for any two values of the studied parameters $\alpha_{g}, \alpha_{m}, M_{0}, N$, a viable set can be found. We note that larger values for $M_{0}$ and negative values for $\alpha_{g}$ are favored. Smaller $M_{0}$ requires small $\alpha_{g}$ in order to have the Higgs mass in the allowed range.

In Figs. 2 and 3 we have plotted the first and the third generation scalar mass parameters and the gaugino mass parameters as a function of $\alpha_{g}$ for $N=3$. We note that the difference of the mass scale of $m_{\tilde{e}_{1}}$ and $m_{\tilde{L}_{1}}$ to the rest of the scalars is a good indicator for the magnitude of $\alpha_{g}$, with large difference implying a larger absolute value for $\alpha_{g}$. For $\alpha_{m}=1$ and $M_{0}=2 \mathrm{TeV}$ only $\alpha_{g}$ close to -1 is allowed by the Higgs mass limit (represented here by the vertical dashed line). In such a case the squark masses turn out to be several TeVs while slepton masses can be of the order of one TeV. Similarly, the ratios of the gaugino mass parameters $M_{3}$ to $M_{1}$ and $M_{3}$ to $M_{2}$ correspond the value of $\alpha_{g}$ with a large ratios implying a $\alpha_{g}$ close to -1 .

\section{RENORMALIZATION GROUP INVARIANTS IN DEFLECTED MIRAGE MEDIATION}

Renormalization group invariants are linear combinations of the soft mass parametersq that remain constant under one-loop renormalization group running. Complete renormalization group invariants for the MSSM together with corresponding sum rules have been derived in 20. The RGIs are derived under several assumptions, including the vanishing of first and second generation Yukawas 20. We have derived the invariants for general $b_{i}$ in order to determine their values above the messenger scale(Table I). Since in deflected mirage mediation new particles are introduced at a certain renormalization scale and integrated out below, one should verify whether the RGIs are affected by the modified spectrum. By considering the renormalization group beta functions (with $\beta(p) \equiv 16 \pi^{2} \frac{d p}{d t}$ and $t=\log \left(\mu / \mu_{0}\right)$ ) for the gauginos and for the coupling constants $(a=1,2,3)$,

$$
\begin{aligned}
\beta\left(g_{a}\right) & =b_{a} g_{a}^{3}, \\
\beta\left(M_{a}\right) & =2 b_{a} g_{a}^{2} M_{a}
\end{aligned}
$$

and by noting that $\beta\left(\frac{M_{r}}{g_{r}^{2}}\right)=0$, we can define a quantity that is constant under renormalization group evolution,

$$
I_{B_{r}} \equiv \frac{M_{r}}{g_{r}^{2}}
$$

Similarly from the full set of MSSM renormalization group equations one can define 12 other invariants that we have enumerated in Tables I and II. If the messenger fields would not enter the theory at an energy scale different of the GUT-scale, the invariants would remain unchanged at all scales. Since the appearance of the messengers modifies the beta functions and contributes to gaugino and scalar masses at the messenger scale $\mu_{\text {mess }}$, the invariant can have a different value above and below the scale.

Just above the messenger scale the value is equal to the GUT-scale value

$$
I_{B_{r}}\left(\mu_{\mathrm{mess}}^{+}\right)=\frac{M_{r}\left(\mu_{\mathrm{mess}}^{+}\right)}{g_{r}^{2}\left(\mu_{\mathrm{mess}}\right)}=I_{B_{r}}\left(\mu_{\mathrm{GUT}}\right) .
$$

We define $\mu_{\text {mess }}^{+}$as evaluation at $\mu_{\text {mess }}$ with modified coefficients $b_{a}^{\prime}$ and without the threshold corrections added to gaugino and scalar masses, and $\mu_{\text {mess }}^{-}$as evaluation at $\mu_{\text {mess }}$ with the usual MSSM coefficients $b_{a}$ and with 


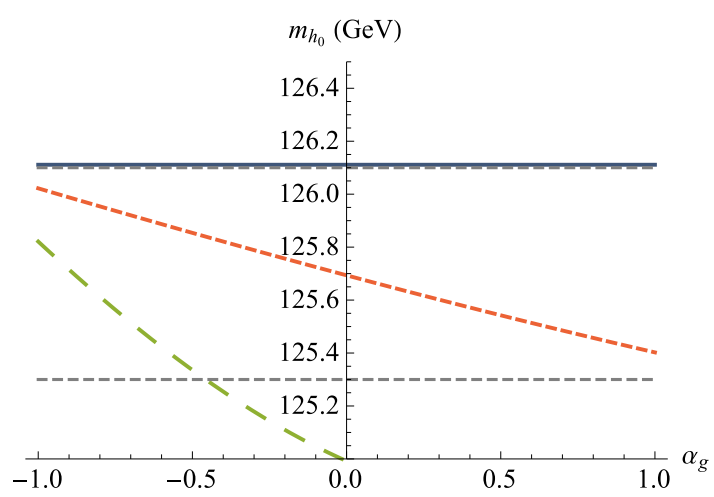

(a) $\alpha_{m}=0.5, M_{0}=3 \mathrm{TeV}$

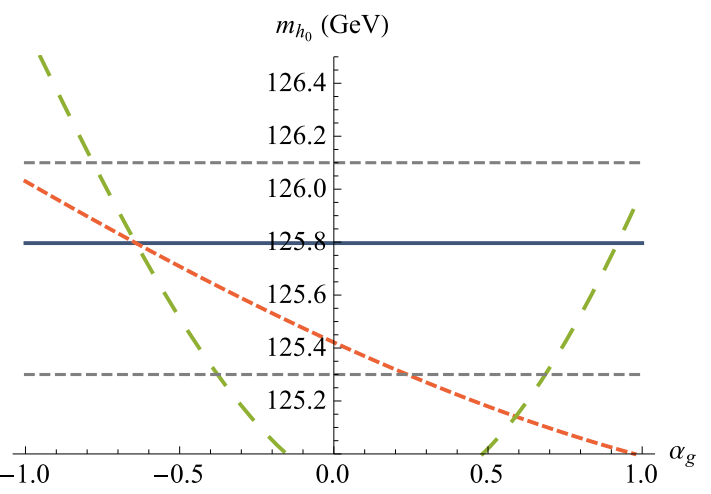

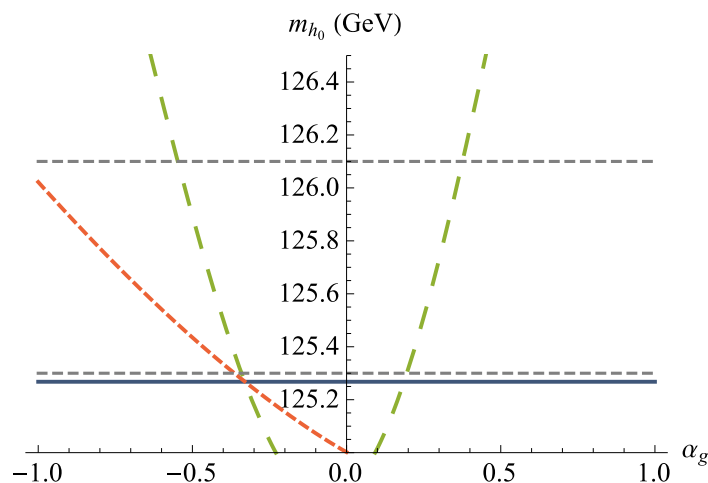

(c) $\alpha_{m}=2, M_{0}=3 \mathrm{TeV}$

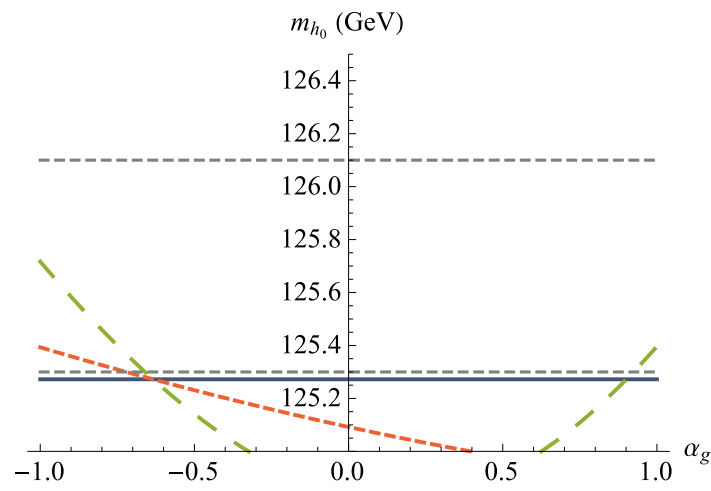

(e) $\alpha_{m}=1, M_{0}=2 \mathrm{TeV}$ (b) $\alpha_{m}=1, M_{0}=3 \mathrm{TeV}$

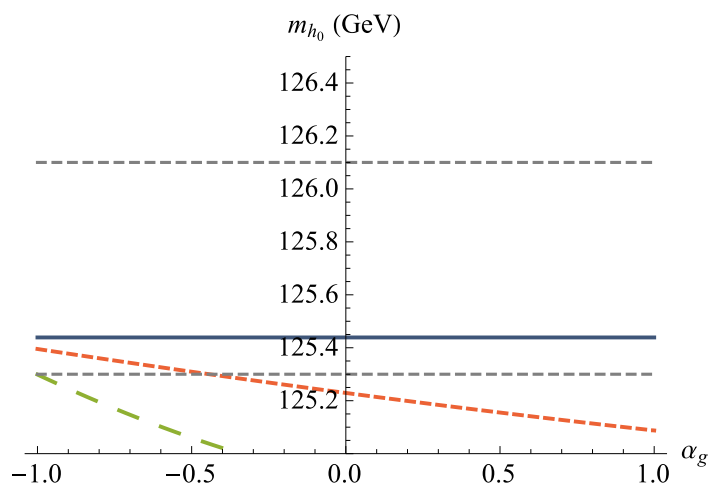

(d) $\alpha_{m}=0.5, M_{0}=2 \mathrm{TeV}$

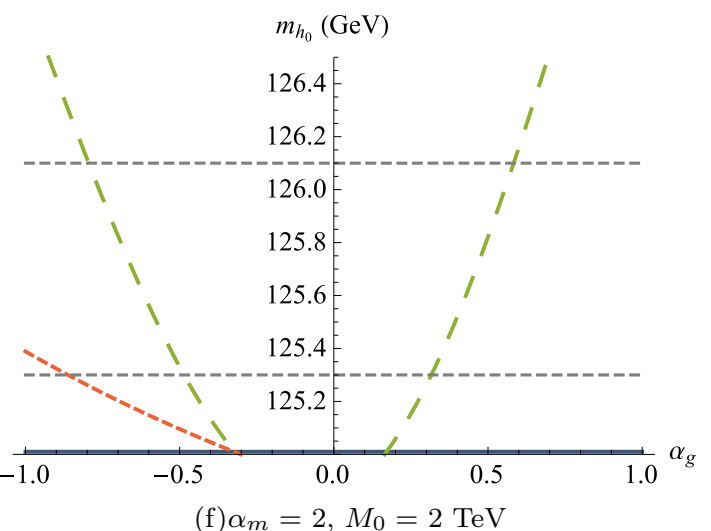

FIG. 1: Higgs mass as a function of $\alpha_{g} \cdot n_{Q}=n_{U}=n_{D}=n_{L}=n_{E}=1 / 2$, and $\mu_{\text {mess }}=10^{12} \mathrm{GeV}$. Thick lines in the order of increasing dash length correspond to $\mathrm{N}=0$ (solid), 3, 10. The Higgs mass is within current experimental limits between the dashed horizontal lines.

threshold corrections added. Below the messenger scale the gaugino masses receive the threshold correction (II.10). Consequently just below the messenger scale

$$
I_{B_{r}}\left(\mu_{\mathrm{mess}}^{-}\right)=\frac{M_{r}\left(\mu_{\mathrm{mess}}^{-}\right)}{g_{r}^{2}\left(\mu_{\mathrm{mess}}\right)}=\frac{M_{r}\left(\mu_{\mathrm{mess}}^{+}\right)}{g_{r}^{2}\left(\mu_{\mathrm{mess}}\right)}+\frac{\Delta M_{r}}{g_{r}^{2}\left(\mu_{\mathrm{mess}}\right)}=I_{B_{r}}\left(\mu_{\mathrm{GUT}}\right)+\Delta I_{B_{r}},
$$

where $\Delta M_{r}$ is as in (II.10) and we have defined

$$
\Delta I_{B_{r}} \equiv \Delta M_{r} / g_{r}^{2}\left(\mu_{\mathrm{mess}}\right)=-N M_{0} /\left(16 \pi^{2}\right)\left(1+\alpha_{g}\right) \alpha_{m} \ln \frac{M_{P}}{m_{3 / 2}} .
$$




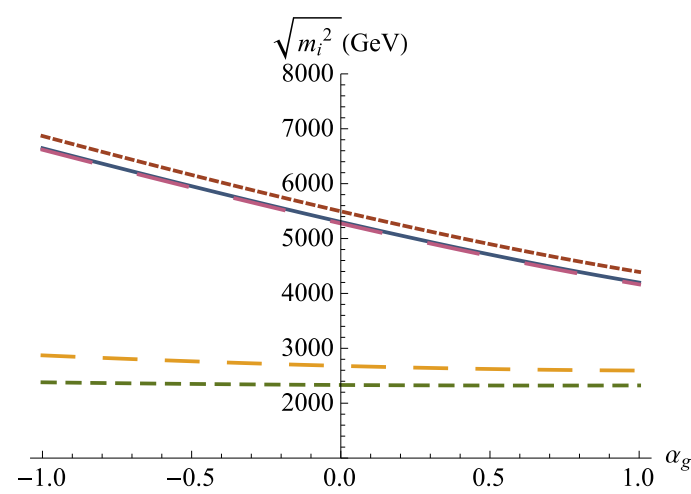

(a)1. generation. $\alpha_{m}=0.5, M_{0}=3 \mathrm{TeV}$

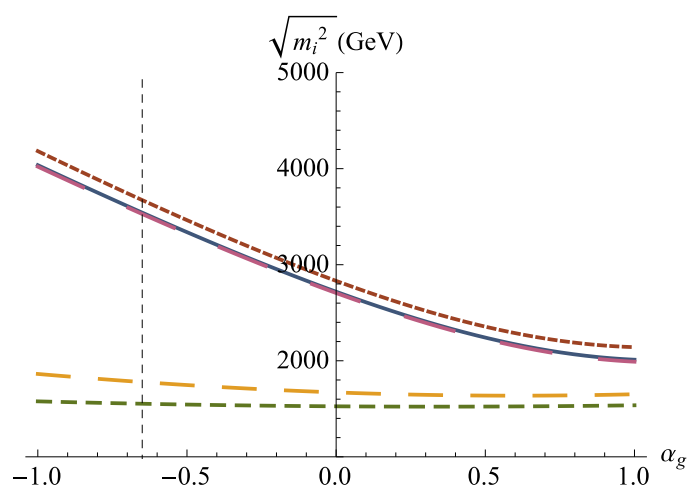

(c)1. generation. $\alpha_{m}=1.0, M_{0}=2 \mathrm{TeV}$

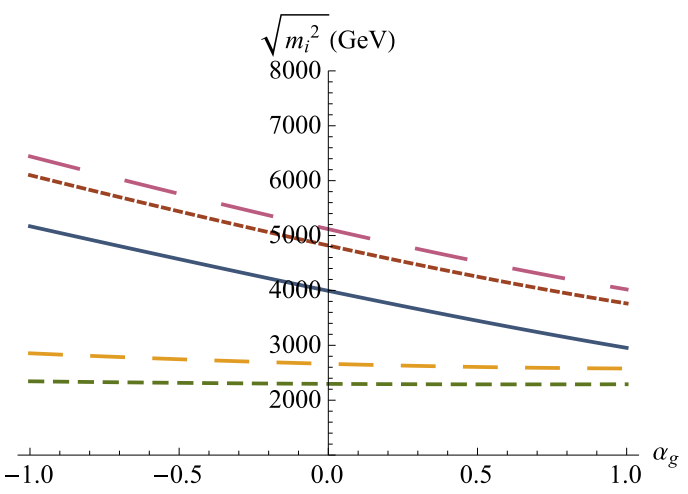

(b)3. generation. $\alpha_{m}=0.5, M_{0}=3 \mathrm{TeV}$

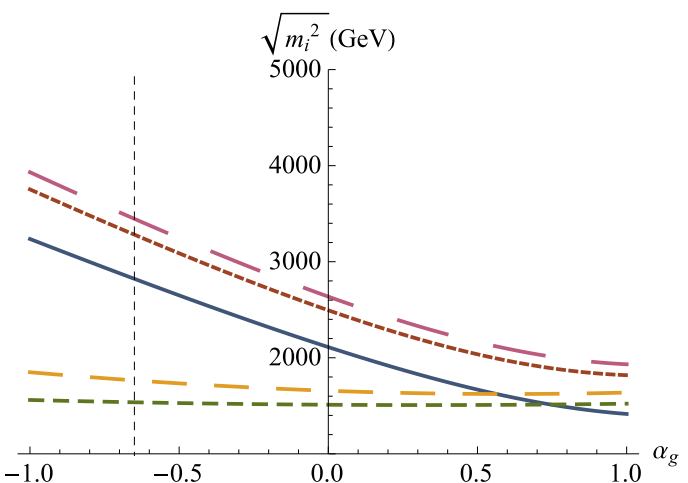

(d)3. generation. $\alpha_{m}=1.0, M_{0}=2 \mathrm{TeV}$

FIG. 2: The first and the third generation soft scalar mass parameters plotted as a function of $\alpha_{g}$. In the order of increasing dash length: $m_{\tilde{U}}$ (solid), $m_{\tilde{Q}_{1}}, m_{\tilde{e}}, m_{\tilde{L}}$, and $m_{\tilde{d}} \cdot n_{Q}=n_{U}=n_{D}=n_{L}=n_{E}=1 / 2, \mu_{\text {mess }}=10^{12} \mathrm{GeV}$, and $\mathrm{N}=3$. In (c) and (d) Higgs mass is in the allowed range on the left hand side of the dashed vertical line.
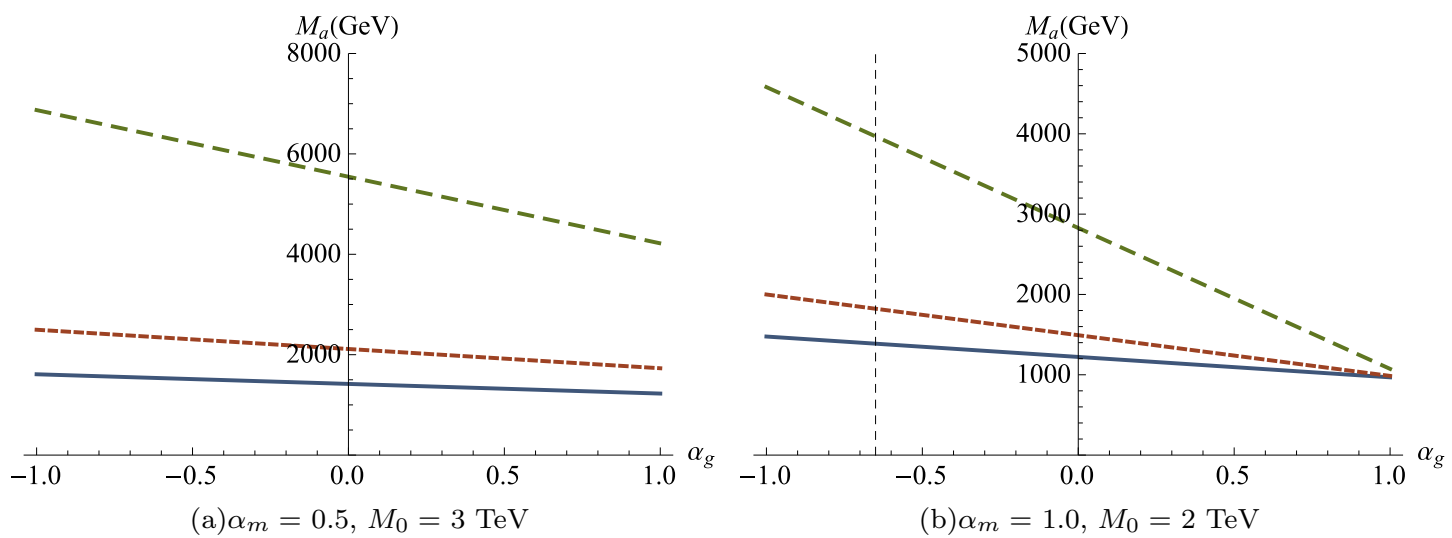

FIG. 3: The gaugino masses plotted as a function of $\alpha_{g}$. We have set $\mu_{\text {mess }}=10^{12} \mathrm{GeV}, n_{Q}=n_{U}=n_{D}=n_{L}=n_{E}=1 / 2$, and $\mathrm{N}=3$. In the order of increasing dash length the lines correspond to $M_{1}$ (solid), $M_{2}, M_{3}$. In (a) Higgs mass is in the allowed range on the left hand side of the dashed horizontal line.

We evolve the couplings down from the GUT scale and obtain

$$
\begin{array}{r}
g_{1}\left(\mu_{\text {mess }}\right)=\frac{2 \sqrt{10} \pi}{\sqrt{40 \pi^{2} / g^{2}+(33+5 N) t_{\text {mess }}}}, \\
g_{2}\left(\mu_{\text {mess }}\right)=\frac{2 \sqrt{2} \pi}{\sqrt{8 \pi^{2} / g^{2}+(1+N) t_{\text {mess }}}},
\end{array}
$$




$$
g_{3}\left(\mu_{\mathrm{mess}}\right)=\frac{2 \sqrt{2} \pi}{\sqrt{8 \pi^{2} / g^{2}+(N-3) t_{\mathrm{mess}}}}
$$

where $t_{\text {mess }}=\ln \mu_{\mathrm{GUT}} / \mu_{\text {mess }}$. Thus while the invariants remain invariant from messenger scale to GUT scale as well as from the eletroweak scale to messenger scale, there is a discontinuity at the messenger scale, which must be taken into account, unless the threshold contributions are cancelled out.

The invariants designated $D_{I}$ are linear combinations of the squared scalar masses with the GUT-scale value of the from

$$
D_{I}=\gamma_{I} m_{3 / 2}^{2}+\delta_{I} M_{0}^{2}
$$

The invariants are constructed in such a way that threshold corrections (II.11) cancel at the messenger scale, thus $D_{I}\left(\mu_{\mathrm{TeV}}\right)=D_{I}\left(\mu_{\mathrm{GUT}}\right)$, and they are "true" invariants in models that include gauge mediated supersymmetry breaking such as the deflected mirage mediation. We present $D_{\chi_{1}}$ as an example with the GUT-scale value

$$
D_{\chi_{1}} \equiv 3\left[3 m_{\tilde{d}_{1}}^{2}-2\left(m_{\tilde{Q}_{1}}^{2}-m_{\tilde{L}_{1}}^{2}\right)-m_{\tilde{u}_{1}}^{2}\right]-m_{\tilde{e}_{1}}^{2}=M_{0}^{2}\left(5+3 n_{U}-9 n_{D}-6 n_{L}+n_{E}+6 n_{Q}\right) .
$$

We use the scalar mass based invariants $D_{I}$ to derive the high energy parameters of the deflected mirage mediation in terms of the scalar masses. The three invariants $I_{M_{a}}$ are linear combinations of the squares of both scalar and gaugino masses, and are also explicitly dependent on $b_{a}$, e.g.

$$
I_{M_{1}}=M_{1}^{2}-\frac{5 b_{1}}{8}\left(m_{\tilde{d}_{1}}^{2}-m_{\tilde{u}_{1}}^{2}-m_{\tilde{e}_{1}}^{2}\right)
$$

where $b_{1}$ is replaced by $b_{1}^{\prime}=b_{1}+N$ above the messenger scale. Thus the shift at the messenger scale has a complex form,

$$
\begin{aligned}
\Delta I_{M_{1}} & \equiv I_{M_{1}}\left(\mu_{\text {mess }}^{+}\right)-I_{M_{1}}\left(\mu_{\text {mess }}^{-}\right) \\
& =\left(M_{1}+\Delta M_{1}\right)^{2}-\frac{5 b_{1}}{8}\left(\left(m_{\tilde{d}_{1}}^{2}+\Delta m_{\tilde{d}_{1}}^{2}\right)-\left(m_{\tilde{u}_{1}}^{2}+\Delta m_{\tilde{u}_{1}}^{2}\right)-\left(m_{\tilde{e}_{1}}^{2}+\Delta m_{\tilde{e}_{1}}^{2}\right)\right)-M_{1}^{2}+\frac{5 b_{1}^{\prime}}{8}\left(m_{\tilde{d}_{1}}^{2}-m_{\tilde{u}_{1}}^{2}-m_{\tilde{e}_{1}}^{2}\right) \\
& =-2 M_{1} \Delta M_{1}-\Delta M_{1}^{2}+\frac{5 N}{8}\left(m_{\tilde{d}_{1}}^{2}-m_{\tilde{u}_{1}}^{2}-m_{\tilde{e}_{1}}^{2}\right)-\frac{5 b_{1}}{8}\left(\Delta m_{\tilde{d}_{1}}^{2}-\Delta m_{\tilde{u}_{1}}^{2}-\Delta m_{\tilde{e}_{1}}^{2}\right)
\end{aligned}
$$

where the gaugino mass and the scalar squareds are evaluated at $\mu_{\text {mess }}^{+}$and $\Delta M_{r}$ and $\Delta m_{i}$ are defined in (II.10) and (II.11). Since $\Delta I_{M_{i}}$ depends on masses at the messenger scale, accessing GUT-scale values from the TeV scale measurements is not as straightforward as with $D_{I}$. As with other invariants, $\Delta I_{M_{r}}$ is generated by the messengers and vanishes when the messengers are removed with $N=0$.

We have listed the correction at the messenger scale and the value at the GUT-scale for each invariant in Table I, except for the $D_{I}$ invariants which are listed in Table II. Fig. 4. shows the values of the invariants $I_{B_{a}}$ and the square roots of $I_{M_{a}}$, and $D_{I}$ above and below the messenger scale at the point $M_{0}=3 \mathrm{TeV}, N=3$, $\alpha_{m}=1, \alpha_{g}=-0.5, t_{\mathrm{MESS}}=-10, n_{u}=1 / 2$, and $n_{h}=1$.

\section{A. Solving parameters using the invariants.}

We will now attempt to utilize the RGIs to solve high scale parameters $m_{3 / 2}, M_{0}$, the messenger scale $\mu_{\text {mess }}$, and the number of messenger pairs $N$ in terms of low energy masses and mass parameters.

Following III.5 we can write three equations involving the invariants $I_{B_{r}}$ by setting the low energy scale value of $I_{B_{r}}$ equal to the value at the GUT-scale corrected by the difference at the messenger scale,

$$
I_{B_{a}}\left(\mu_{\mathrm{GUT}}\right)=I_{B_{a}}\left(\mu_{\mathrm{TeV}}\right)-\Delta I_{B_{a}},
$$

where $I_{B_{r}}$ is defined in (III.3) and $\Delta I_{B_{r}}$ in (III.6). We note that $\Delta I_{B_{r}}$ vanishes if $\alpha_{g}=-1$. Thus the equivalence of the TeV-scale value of $I_{B_{a}}$ to its GUT-scale value cannot be taken as proof of the absence of gauge messengers.

The equations III.12 provide three independent solutions for $m_{3 / 2}$, and $\alpha_{g}$, which we distinguish from each 


\begin{tabular}{|c|l|l|l|}
\hline Invariant & Definition & Correction at the messenger scale & Value at the GUT scale \\
\hline \hline$I_{B_{r}}$ & $M_{r} / g_{r}^{2}$ & $\Delta M_{r} / g_{r}^{2}$ & $M_{0} / g^{2}+\frac{b_{a}^{\prime}}{16 \pi^{2}} m_{3 / 2}$ \\
\hline$I_{M_{1}}$ & $M_{1}^{2}-\frac{5 b_{1}}{8}\left(m_{\tilde{d}_{1}}^{2}-m_{\tilde{u}_{1}}^{2}-m_{\tilde{e}_{1}}^{2}\right)$ & $\begin{array}{l}-2 M_{1} \Delta M_{1}-\Delta M_{1}^{2}+ \\
\frac{5 N}{8}\left(m_{\tilde{d}_{1}}^{2}-m_{\tilde{u}_{1}}^{2}-m_{\tilde{e}_{1}}^{2}\right) \\
-\frac{5 b_{1}}{80} \Delta m^{2} g_{1}^{4}\end{array}$ & $M_{0}^{2}\left(1+n_{\epsilon_{1}} b_{1}^{\prime}\right)$ \\
\hline$I_{M_{2}}$ & $M_{2}^{2}+\frac{b_{2}}{24}\left(9\left(m_{\tilde{d}_{1}}^{2}-m_{\tilde{u}_{1}}^{2}\right)+16 m_{\tilde{L}_{1}}^{2}-m_{\tilde{e}_{1}}^{2}\right)$ & $\begin{array}{l}\frac{N}{24}\left(9\left(m_{\tilde{d}_{1}}^{2}-m_{\tilde{u}_{1}}^{2}\right)+16 m_{\tilde{L}_{1}}^{2}-m_{\tilde{e}_{1}}^{2}\right) \\
+\frac{3 b_{2}}{48} \Delta m^{2} g_{2}^{4}\end{array}$ & $M_{0}^{2}\left(1+n_{\epsilon_{2}} b_{2}^{\prime}\right)$ \\
\hline$I_{M_{3}}$ & $M_{3}^{2}+\frac{b_{3}}{16}\left(5 m_{\tilde{d}_{1}}^{2}+m_{\tilde{u}_{1}}^{2}-m_{\tilde{e}_{1}}^{2}\right)$ & $\begin{array}{l}2 M_{3} \Delta M_{3}+\Delta M_{3}^{2}- \\
\frac{N}{16}\left(5 m_{\tilde{d}_{1}}^{2}+m_{\tilde{u}_{1}}^{2}-m_{\tilde{e}_{1}}^{2}\right) \\
+\frac{b_{3}}{16} \Delta m^{2} g_{3}^{4}\end{array}$ & \\
\hline$I_{g_{3}}$ & $1 / g_{1}^{2}-\left(b_{1} / b_{3}\right) g_{3}^{-2}$ & $28 N /\left(5 g_{2}^{2}(1+N)\right)$ & $1 / g^{2}\left(1-b_{1}^{\prime} / b_{3}^{\prime}\right)$ \\
\hline & $1 / g_{1}^{2}-\left(b_{1} / b_{2}\right) g_{2}^{-2}$ & $-16 N /\left(5 g_{3}^{2}(3-N)\right)$ & $1 / g^{2}\left(1-b_{1}^{\prime} / b_{2}^{\prime}\right)$ \\
\hline
\end{tabular}

TABLE I: The renormalization group invariants $I_{A}$ involving scalar masses, gaugino masses and coupling constants. The second column defines the invariant in terms of soft masses and couplings without messenger fields present. The third column describes the difference of the value of the invariant above and below messenger scale; the masses and the couplings are to be evaluated at the messenger scale. The fourth column describes the value of the invariant at the GUT scale; the couplings are to be evaluated at the GUT scale. The quantity $\Delta m^{2}$ is defined as $N /\left(16 \pi^{4}\right)\left(M_{0} \alpha_{m}\left(1+\alpha_{g}\right) \ln \frac{M_{P}}{m_{3 / 2}}\right)^{2}$. The combinations of modular weights $n_{\epsilon_{a}}$ are defined in III.69-2III.71.

other by designating with the subindex $(a)$,

$$
\begin{aligned}
& m_{3 / 2(a)}=16 \pi^{2} \frac{\sum_{b, c=1}^{3} \epsilon_{a b c} I_{B_{c}}}{\sum_{d, e=1}^{3} \epsilon_{a d e} b_{e}}, \quad(a=1,2,3), \\
& \alpha_{g_{(a)}}=\frac{b_{a} g^{2} m_{3 / 2}-16 \pi^{2} g^{2} I_{B_{a}}+16 \pi^{2} M_{0}}{g^{2} m_{3 / 2} N}, \quad(a=1,2,3) .
\end{aligned}
$$

It is easy to verify by evolving $g_{i}$ that the deflected mirage mediation coupling constant at the GUT scale, $g$, is related to $g_{\mathrm{GUT}}$, which is the coupling constant at the GUT-scale with $N=0$, by

$$
\frac{1}{g}=\sqrt{\frac{1}{g_{\mathrm{GUT}}^{2}}-\frac{N t_{\mathrm{mess}}}{8 \pi^{2}}} .
$$

A different set of parameters to eliminate could, of course, be chosen, but this choice proves to be most convenient for solving all parameters, as $M_{0}$ is readily solved from the scalar mass involving invariants $D_{I}$ which do not allow the determination of $\alpha_{g}$.

The invariants $D_{\chi_{1}}, D_{B_{13}}, D_{Y_{13} H}, D_{Z}$, and $D_{Y} \alpha$ are composed of linear combinations of soft scalar mass parameters and have identical values above and below the messenger scale as the linear combinations of the mass parameters are chosen so that the threshold corrections (II.11) cancel out. The invariants have the schematic form

$$
D_{I}=\gamma_{I} m_{3 / 2}^{2}+\delta_{I} M_{0}^{2}
$$




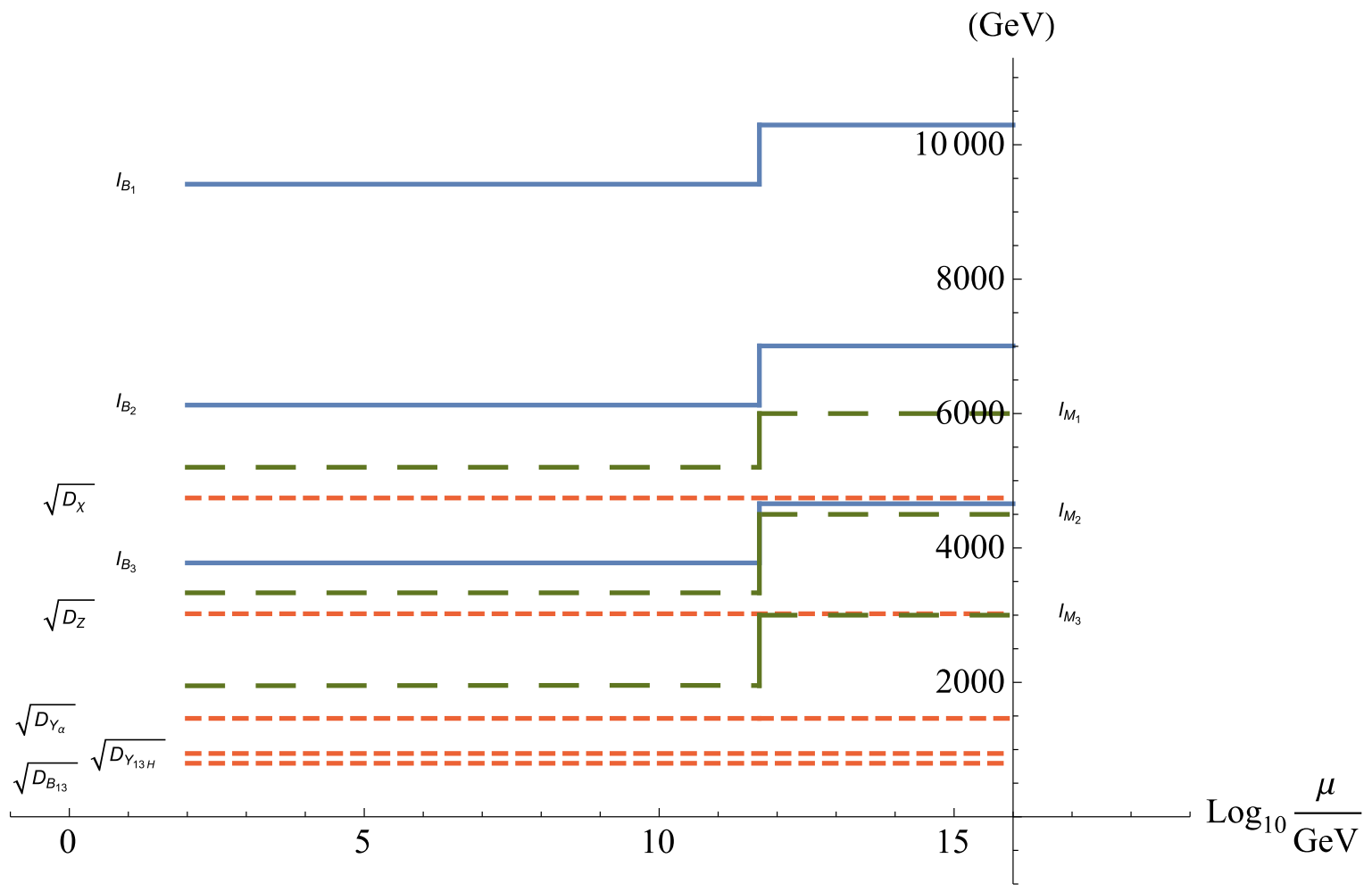

FIG. 4: The renormalization group invariants $I_{B_{a}}$ (blue, solid) and the square roots of the absolute value of the invariants $D_{I}$ (red, small dash) and $I_{M_{a}}$ (green, large dash). Here $n_{u}=1 / 2, n_{h}=, M_{0}=3 \mathrm{TeV} \alpha_{m}=1, \alpha_{g}=-0.5, \mu_{\mathrm{MESS}}=10^{12}$ $\mathrm{GeV}$, and $N=3$. $D_{L_{13}}=0$ is not shown.

\begin{tabular}{|c|c|c|c|}
\hline I & Definition & $\gamma_{I}$ & $\delta_{I}$ \\
\hline \hline$D_{Z}$ & $3\left(m_{\tilde{d}_{3}}^{2}-m_{\tilde{d}_{1}}^{2}\right)+2\left(m_{\tilde{L}_{3}}^{2}-m_{H_{d}}^{2}\right)$ & $Y_{Z a}+Y_{Z b} g^{2}$ & $2 n_{\alpha}$ \\
\hline$D_{\chi_{1}}$ & $3\left(3 m_{\tilde{d}_{1}}^{2}-2\left(m_{\tilde{Q}_{1}}^{2}-m_{\tilde{L}_{1}}^{2}\right)-m_{\tilde{u}_{1}}^{2}\right)-m_{\tilde{e}_{1}}^{2}$ & 0 & $n_{\beta}$ \\
\hline$D_{L_{13}}$ & $2\left(m_{\tilde{L}_{1}}^{2}-m_{\tilde{L}_{3}}^{2}\right)-m_{\tilde{e}_{1}}^{2}+m_{\tilde{e}_{3}}^{2}$ & 0 & 0 \\
\hline$D_{B_{13}}$ & $2\left(m_{\tilde{Q}_{1}}^{2}-m_{\tilde{Q}_{3}}^{2}\right)-m_{\tilde{u}_{1}}^{2}+m_{\tilde{u}_{3}}^{2}-m_{\tilde{d}_{1}}^{2}+m_{\tilde{d}_{3}}^{2}$ & $Y_{B_{13} a}+Y_{B_{13} b} g^{2}$ & 0 \\
\hline$D_{Y_{13 H}}$ & $-\frac{10}{13}\left(m_{\tilde{Q}_{3}}^{2}-2 m_{\tilde{u}_{3}}^{2}+m_{\tilde{d}_{3}}^{2}-m_{\tilde{\tilde{L}}_{3}}^{2}+m_{\tilde{e}_{3}}^{2}+m_{H_{u}}^{2}-m_{H_{d}}^{2}\right)$ & $\frac{10}{13}\left(-Y_{\alpha 1}-Y_{\alpha 2} g^{2}\right)$ & $-\frac{1}{13} n_{\gamma}$ \\
\hline$D_{Y} \alpha$ & $\left(m_{H_{u}}^{2}-m_{H_{d}}^{2}+\sum_{g e n}\left(m_{\tilde{Q}_{\tilde{U}}}^{2}-2 m_{\tilde{u}}^{2}+m_{\tilde{d}_{\tilde{d}}}^{2}-m_{\tilde{L}_{1}}^{2}+m_{\tilde{e}}^{2}\right)\right) / g^{2}$ & $\frac{1}{g^{2}}\left(Y_{\alpha 1}+Y_{\alpha 2}\right)$ & $-\frac{1}{g^{2}} n_{\delta}$ \\
\hline
\end{tabular}

TABLE II: The invariants $D_{I}$ and their GUT-scale values parametrised as $D_{I}\left(\mu_{\mathrm{GUT}}\right)=\gamma_{I} m_{3 / 2}^{2}+\delta_{I} M_{0}^{2}$. See $[$ III.20]III.28. 
at the GUT-scale, where the coefficients $\gamma_{I}, \delta_{I}, \epsilon_{I}$, are determined by the Yukawa couplings $y_{\tau}, y_{t}$ and $y_{b}$ and $g$ at the GUT scale We have listed the values in Table II, where

$$
\begin{aligned}
n_{\alpha} & \equiv n_{H_{d}}-n_{L}, \\
n_{\beta} & \equiv 5+3 n_{U}-9 n_{D}-6 n_{L}+n_{E}+6 n_{Q}, \\
n_{\gamma} & \equiv 3 n_{D}+3 n_{E}+10 n_{H_{d}}-10 n_{H_{u}}-3 n_{L}+3 n_{Q}-6 n_{U}, \\
n_{\delta} & \equiv 3 n_{D}+3 n_{E}-n_{H_{d}}+n_{H_{u}}-3 n_{L}+3 n_{Q}-6 n_{U}, \\
n_{\epsilon} & \equiv n_{H u}+n_{Q}+n_{U}-3, \\
Y_{B_{13} a} \equiv & \frac{1}{10240 \pi^{4}}\left[1440 y_{b}^{6}+240 y_{b}^{4} y_{\tau}^{2}+240 y_{b}^{4} y_{t}^{2}-1440 y_{b}^{4}-240 y_{b}^{2} y_{\tau}^{2}+240 y_{b}^{2} y_{t}^{4}-480 y_{b}^{2} y_{t}^{2}\right. \\
& \left.+1440 y_{t}^{6}-1440 y_{t}^{4}\right], \\
Y_{B_{13} b} \equiv & \frac{1}{30720 \pi^{4}}\left[-2105 y_{b}^{4}+2105 y_{b}^{2}-2208 y_{t}^{4}+2208 y_{t}^{2}\right], \\
Y_{Z a} \equiv & \frac{1}{10240 \pi^{4}}\left[1440 y_{b}^{6}+240 y_{b}^{4} y_{\tau}^{2}+240 y_{b}^{4} y_{t}^{2}+1440 y_{b}^{4}-720 y_{b}^{2} y_{\tau}^{2}-240 y_{b}^{2} y_{t}^{2}-640 y_{\tau}^{4}\right], \\
Y_{Z b} \equiv & \frac{1}{10240 \pi^{4}}\left[-2105 y_{b}^{4}+2105 y_{b}^{2}+768 y_{\tau}^{2}\right], \\
Y_{\alpha 1} \equiv & \frac{1}{30720 \pi^{4}}\left[1440 y_{b}^{6}+240 y_{b}^{4} y_{\tau}^{2}+240 y_{b}^{4} y_{t}^{2}-1440 y_{b}^{4}-960 y_{b}^{2} y_{\tau}^{2}-480 y_{b}^{2} y_{t}^{4}\right. \\
& \left.+240 y_{b}^{2} y_{t}^{2}-960 y_{\tau}^{4}-2880 y_{t}^{6}+2880 y_{t}^{4}\right], \\
Y_{\alpha 2} \equiv & \frac{1}{30720 \pi^{4}}\left[-2105 y_{b}^{4}+2105 y_{b}^{2}+1152 y_{\tau}^{2}+4416 y_{t}^{4}-4416 y_{t}^{2}\right] .
\end{aligned}
$$

The parameter $M_{0}$ is easily solved using the GUT-scale value of $D_{\chi_{1}}$,

$$
D_{\chi_{1}}=3\left(3 m_{\tilde{d}_{1}}^{2}-2\left(m_{\tilde{Q}_{1}}^{2}-m_{\tilde{L}_{1}}^{2}\right)-m_{\tilde{u}_{1}}^{2}\right)-m_{\tilde{e}_{1}}^{2}=n_{\beta} M_{0},
$$

to obtain

$$
M_{0}=\sqrt{\frac{D_{\chi_{1}}}{n_{\beta}}}, \text { for } n_{\beta} \neq 0,
$$

For solving $g$ we use the GUT-scale values of the two invariants $D_{Y_{13 H}}, D_{Y} \alpha$, defined as

$$
\begin{aligned}
D_{Y_{13 H}} & =m_{\tilde{Q}_{1}}^{2}-2 m_{\tilde{u}_{1}}^{2}+m_{\tilde{d}_{1}}^{2}-m_{\tilde{L}_{1}}^{2}+m_{\tilde{e}_{1}}^{2}-\frac{10}{13}\left(m_{\tilde{Q}_{3}}^{2}-2 m_{\tilde{u}_{3}}^{2}+m_{\tilde{d}_{3}}^{2}-m_{\tilde{L}_{3}}^{2}+m_{\tilde{e}_{3}}^{2}+m_{H_{u}}^{2}-m_{H_{d}}^{2}\right) \\
& =\frac{10}{13}\left(-Y_{\alpha 1}-Y_{\alpha 2} g^{2}\right) m_{3 / 2}^{2}-\frac{1}{13} n_{\gamma} M_{0}^{2}, \\
D_{Y} \alpha & =\left(m_{H_{u}}^{2}-m_{H_{d}}^{2}+\sum_{g e n}\left(m_{\tilde{Q}}^{2}-2 m_{\tilde{u}}^{2}+m_{\tilde{d}}^{2}-m_{\tilde{L}}^{2}+m_{\tilde{e}}^{2}\right)\right) / g^{2}=\frac{1}{g^{2}}\left(Y_{\alpha 1}+g^{2} Y_{\alpha 2}\right) m_{3 / 2}^{2}-\frac{1}{g^{2}} n_{\delta} M_{0}^{2},
\end{aligned}
$$

Yukawa-dependent terms $Y_{\alpha 1}$ and $Y_{\alpha 1}$ can be eliminated by forming the linear combination

$$
D_{Y_{13 H}}+\frac{10 g^{2}}{13} D_{Y \alpha}=-\frac{10}{13} M_{0}^{2}\left(n_{\delta}+n_{\gamma}\right) .
$$

We obtain the solution

$$
g^{2}=-\frac{\mathcal{Y}\left(n_{\delta \gamma}\right)}{10 D_{Y \alpha}}
$$

where we have defined

$$
\begin{aligned}
& n_{\delta \gamma}=n_{\delta}+n_{\gamma}=2 n_{\gamma}-11 n_{H_{d}}+11 n_{H_{d}}, \\
& \mathcal{Y}\left(n_{A}\right)=13 D_{Y_{13 H}}+10 M_{0}^{2} n_{A} .
\end{aligned}
$$


Using the solution III.30 for $M_{0}$, we write

$$
\begin{aligned}
& g^{2}=\frac{-10 D_{\chi_{1}}\left(n_{\delta}+n_{\gamma}\right)-13 D_{Y_{13 H}} n_{\beta}}{10 D_{Y \alpha} \sqrt{n_{\beta}}}, \\
& \mathcal{Y}\left(n_{A}\right)=13 D_{Y_{13 H}}+\frac{10 n_{A}}{n_{\beta}} D_{\chi_{1}} .
\end{aligned}
$$

Since from (III.16) we have

$$
t_{\mathrm{MESS}}=\frac{8 \pi^{2}\left(g^{2}-g_{\mathrm{GUT}}^{2}\right)}{g^{2} g_{\mathrm{GUT}}^{2} N},
$$

the relation of $t_{\text {MESS }}$ and $N$ is fixed once $g^{2}$ is measured. In addition to III.34, equations

$$
\left\{\begin{array}{l}
D_{Y_{13 H}}=\frac{10}{13}\left(-Y_{\alpha 1}-Y_{\alpha 2} g^{2}\right) m_{3 / 2}^{2}-\frac{1}{13} n_{\gamma} M_{0}^{2} \\
D_{Y \alpha}=\frac{1}{g^{2}}\left(Y_{\alpha 1}+Y_{\alpha 2}\right) m_{3 / 2}^{2}-\frac{1}{g^{2}} n_{\delta} M_{0}^{2}
\end{array}\right.
$$

produce a solution to $m_{3 / 2}$,

$$
m_{3 / 2}=\frac{32 \sqrt{30} \pi^{2} \sqrt{D_{Y \alpha} \mathcal{Y}\left(n_{\gamma}\right)}}{\sqrt{Y_{\alpha 2} \mathcal{Y}\left(n_{\delta \gamma}\right)-10 D_{Y \alpha} Y_{\alpha 1}}} .
$$

By solving simultaneously the equations

$$
\left\{\begin{array}{l}
D_{B_{13}}=\left(Y_{B_{13} a}+Y_{B_{13} b} g^{2}\right) m_{3 / 2}^{2} \\
D_{Z}=2 n_{\alpha} M_{0}^{2}+\left(Y_{Z a}+Y_{Z b} g^{2}\right) m_{3 / 2}^{2}
\end{array},\right.
$$

we find alternative solutions to $\mathcal{M}_{0}$ and $m_{3 / 2}$ that are dependent on the Yukawa couplings

$$
\begin{aligned}
m_{3 / 2}= & \frac{32 \sqrt{30} \sqrt{D_{B_{13}}}}{\sqrt{g^{2} Y_{B_{13} b}+Y_{B_{13} a}}} \\
& =\frac{320 \sqrt{3} \sqrt{D_{B_{13}}} \sqrt{D_{Y \alpha}} \sqrt{n_{\beta}}}{\sqrt{10 D_{Y \alpha} n_{\beta} Y_{B_{13} a}-Y_{B_{13} b} \mathcal{Y}\left(n_{\delta \gamma}\right)}}, \\
M_{0}= & \frac{\sqrt{3 D_{B_{13}}\left(g^{2} Y_{Z b}+Y_{Z a}\right)-\pi^{4} D_{Z}\left(g^{2} Y_{B_{13} b}+Y_{B_{13} a}\right)}}{\sqrt{2} \pi^{2} \sqrt{-n_{\alpha}\left(g^{2} Y_{B_{13} b}+Y_{B_{13} a}\right)}}= \\
& \sqrt{\frac{\left(\pi^{4} D_{Z} Y_{B_{13} b}-3 D_{B_{13}} Y_{Z b}\right) \mathcal{Y}\left(n_{\delta \gamma}\right)-10 D_{Y \alpha} n_{\beta}\left(\pi^{4} D_{Z} Y_{B_{13} a}-3 D_{B_{13}} Y_{Z a}\right)}{2 \pi^{4} n_{\alpha}\left(Y_{B_{13} b}\left(\mathcal{Y}\left(n_{\delta \gamma}\right)-10 D_{Y \alpha} n_{\beta} Y_{B_{13} a}\right)\right.}} .
\end{aligned}
$$

\section{Yukawas in terms of invariants}

Instead of the mass parameters it is possible to use $D_{I}$ to determine the values of the Yukawas at the GUT scale, although the necessary parameters for running the Yukawas up from the low energy scale are solvable independently of the Yukawas. We assume a small $y_{b}$ and $y_{\tau}$ compared to $y_{t}$ and small enough $y_{t}$ to neglect terms with $y_{t}^{6}$ and higher order, and $y_{b}^{4}, y_{\tau}^{4}$ and higher order. The equations (III.23)-(III.28) are then reduced to

$$
\begin{aligned}
Y_{B_{13} a} & =-1440 y_{t}^{4}, \\
Y_{B_{13} b} & =-2105 y_{b}^{2}-2208 y_{t}^{4}+2208 y_{t}^{2}, \\
Y_{Z a} & =0, \\
Y_{Z b} & =2105 y_{b}^{2}, \\
Y_{\alpha 1} & =2880 y_{t}^{4}, \\
Y_{\alpha 2} & =2105 y_{b}^{2}+1152 y_{\tau}^{2}+4416 y_{t}^{4}-4416 y_{t}^{2} .
\end{aligned}
$$


From (III.42), and (III.44) we can then solve

$$
\begin{aligned}
y_{\tau}= & \frac{4 \sqrt{5} \sqrt{-D_{Y \alpha}} \sqrt{60 D_{B_{13}}-\pi^{4}\left(13 \mathcal{Y}\left(n_{\gamma}\right)-10 D_{Z}+20 M_{0}^{2} n_{\alpha}\right)}}{3 \sqrt{m_{3 / 2}^{2} \mathcal{Y}\left(n_{\delta \gamma}\right)}}, \\
y_{b}= & \frac{64 \sqrt{\frac{5}{421}} \pi^{2} \sqrt{-D_{Y \alpha}\left(D_{Z}-2 M_{0}^{2} n_{\alpha}\right)}}{\sqrt{m_{3 / 2}^{2} \mathcal{Y}\left(n_{\delta \gamma}\right)}}, \\
y_{t}= & \frac{1}{6 m_{3 / 2}^{2}\left(23 \mathcal{Y}\left(n_{\delta \gamma}\right)-150 D_{Y \alpha}\right)}\left\{4761 m_{3 / 2}^{4} \mathcal{Y}\left(n_{\delta \gamma}\right)^{2}+69 \mathcal{Y}\left(n_{\delta \gamma}\right)\right. \\
& \left.+\sqrt{38400 D_{Y \alpha} m_{3 / 2}^{2}\left(3 D_{B_{13}}+\pi^{4}\left(D_{Z}-2 M_{0}^{2} n_{\alpha}\right)\right)\left(23 \mathcal{Y}\left(n_{\delta \gamma}\right)-150 D_{Y \alpha}\right)}\right\} .
\end{aligned}
$$

Note that while the solution $D_{\chi_{1}} / n_{\beta}$ can be substituted for $M_{0}^{2}$, and $16 \pi^{2}\left(I_{B_{1}}-I_{B_{3}}\right) /\left(b_{1}-b_{3}\right)$ for $m_{3 / 2}$ here, the solution (III.46) for $M_{0}$ and the solutions (III.43) and (III.45) for $m_{3 / 2}$ derived above, are not independent from (III.53)-(III.55) and thus cannot be used.

\section{Solving $N$}

For solving $N$ we use the remaining three invariants composed of the mass parameters,

$$
\begin{aligned}
I_{M_{1}} & =M_{1}^{2}-\frac{5 b_{1}^{\prime}}{8}\left(m_{\tilde{d}_{1}}^{2}-m_{\tilde{u}_{1}}^{2}-m_{\tilde{e}_{1}}^{2}\right), \\
I_{M_{2}} & =M_{2}^{2}+\frac{b_{2}^{\prime}}{24}\left(9\left(m_{\tilde{d}_{1}}^{2}-m_{\tilde{u}_{1}}^{2}\right)+16 m_{\tilde{L}_{1}}^{2}-m_{\tilde{e}_{1}}^{2}\right), \\
I_{M_{3}} & =M_{3}^{2}+\frac{b_{3}^{\prime}}{16}\left(5 m_{\tilde{d}_{1}}^{2}+m_{\tilde{u}_{1}}^{2}-m_{\tilde{e}_{1}}^{2}\right) .
\end{aligned}
$$

We remind the reader that parameter $b_{a}^{\prime}=b_{a}+N$ is to be replaced with $b_{a}$ below the messenger scale. In order to connect $\mathrm{TeV}$ scale measurements to the GUT scale parameter values, we examine $I_{M_{1}}$ above the messenger scale:

$$
\begin{aligned}
I_{M_{1}}\left(\mu_{\mathrm{MESS}}^{+}\right) & =M_{1}^{2}\left(\mu_{\mathrm{MESS}}^{+}\right)-\frac{5 b_{1}^{\prime}}{8}\left(m_{\tilde{d}_{1}}^{2}\left(\mu_{\mathrm{MESS}}^{+}\right)-m_{\tilde{u}_{1}}^{2}\left(\mu_{\mathrm{MESS}}^{+}\right)-m_{\tilde{e}_{1}}^{2}\left(\mu_{\mathrm{MESS}}^{+}\right)\right) \\
& =I_{M_{1}}\left(\mu_{\mathrm{GUT}}\right) .
\end{aligned}
$$

By using the relations

$$
\begin{aligned}
& I_{B_{1}}\left(\mu_{\mathrm{GUT}}\right)=I_{B_{1}}\left(\mu_{\mathrm{MESS}}^{+}\right)=M_{1}\left(\mu_{\mathrm{MESS}}^{+}\right) / g_{1}^{4}\left(\mu_{\mathrm{MESS}}\right), \\
& m_{i}^{2}\left(\mu_{\mathrm{MESS}}^{+}\right)=m_{i}^{2}\left(\mu_{\mathrm{MESS}}^{-}\right)-\Delta m_{i}^{2},
\end{aligned}
$$

we eliminate the mass parameters measured above messenger scale and obtain

$$
\begin{aligned}
I_{M_{1}}\left(\mu_{\mathrm{GUT}}\right) & =I_{B_{1}}^{2}\left(\mu_{\mathrm{GUT}}\right) g_{1}^{4}\left(\mu_{\mathrm{MESS}}\right)-\frac{5 b_{1}^{\prime}}{8}\left(m_{\tilde{d}_{1}}^{2}\left(\mu_{\mathrm{MESS}}^{-}\right)-m_{\tilde{u}_{1}}^{2}\left(\mu_{\mathrm{MESS}}^{-}\right)-m_{\tilde{e}_{1}}^{2}\left(\mu_{\mathrm{MESS}}^{-}\right)\right) \\
& +\frac{5 b_{1}^{\prime}}{8}\left(\Delta m_{\tilde{d}_{1}}^{2}+\Delta m_{\tilde{u}_{1}}^{2}-\Delta m_{\tilde{e}_{1}}^{2}\right) .
\end{aligned}
$$

Directly from (III.57) and (III.61) we see that below the messenger scale $5 b_{1} / 8\left(m_{\tilde{d}_{1}}^{2}-m_{\tilde{u}_{1}}^{2}-m_{\tilde{e}_{1}}^{2}\right)=I_{M_{1}}-M_{1}^{2}=$ $I_{M_{1}}-I_{B_{1}} g_{1}^{4}\left(\mu_{\mathrm{MESS}}\right)$. Thus

$$
\begin{aligned}
I_{M_{1}}\left(\mu_{\mathrm{GUT}}\right) & =I_{B_{1}}^{2}\left(\mu_{\mathrm{GUT}}\right) g_{1}^{4}\left(\mu_{\mathrm{MESS}}\right)+\frac{b_{1}^{\prime}}{b_{1}}\left(I_{M_{1}}-I_{B_{1}}^{2} g_{1}^{4}\left(\mu_{\mathrm{MESS}}\right)\right) \\
& +\frac{5 b_{1}^{\prime}}{8}\left(\Delta m_{\tilde{d}_{1}}^{2}+\Delta m_{\tilde{u}_{1}}^{2}-\Delta m_{\tilde{e}_{1}}^{2}\right) .
\end{aligned}
$$


We have dropped the argument $\mu_{\mathrm{MESS}}^{-}$so that the low scale value of the invariants are meant unless otherwise specified. Analoguously

$$
\begin{aligned}
I_{M_{2}}\left(\mu_{\mathrm{GUT}}\right)= & I_{B_{2}}^{2}\left(\mu_{\mathrm{GUT}}\right) g_{2}^{4}\left(\mu_{\mathrm{MESS}}\right)+\frac{b_{1}^{\prime}}{b_{1}}\left(I_{M_{1}}-I_{B_{2}}^{2} g_{2}^{4}\left(\mu_{\mathrm{MESS}}\right)\right) \\
& -\frac{b_{2}^{\prime}}{24}\left(9\left(\Delta m_{\tilde{d}_{1}}^{2}-\Delta m_{\tilde{u}_{1}}^{2}\right)+16 \Delta m_{\tilde{L}_{1}}^{2}-\Delta m_{\tilde{e}_{1}}^{2}\right) \\
I_{M_{3}}\left(\mu_{\mathrm{GUT}}\right) & =I_{B_{3}}^{2}\left(\mu_{\mathrm{GUT}}\right) g_{3}^{4}\left(\mu_{\mathrm{MESS}}\right)+\frac{b_{1}^{\prime}}{b_{1}}\left(I_{M_{3}}-I_{B_{3}}^{2} g_{3}^{4}\left(\mu_{\mathrm{MESS}}\right)\right) \\
& -\frac{b_{3}^{\prime}}{16}\left(5 \Delta m_{\tilde{d}_{1}}^{2}+\Delta m_{\tilde{u}_{1}}^{2}-\Delta m_{\tilde{e}_{1}}^{2}\right) .
\end{aligned}
$$

After substituting the GUT scale values $I_{B_{1}}\left(\mu_{\mathrm{GUT}}\right)$ and $I_{M_{1}}\left(\mu_{\mathrm{GUT}}\right)$, the corrections to the scalar masses II.11) with $\alpha_{g}$ from (III.14, $g_{a}\left(\mu_{\text {MESS }}\right)$ from (III.7), and $\alpha_{g_{(a)}}$, the equations (III.63)-(III.65) can be collectively written as

$$
\begin{aligned}
& 256 \pi^{4}\left\{-b_{a}^{2}\left(g^{2}-g_{\mathrm{GUT}}^{2}\right)^{2}\left(\mathcal{I}_{M_{a}}-M_{0}^{2}\right)+b_{a} g^{2} N\left(g^{2}\left(M_{0}^{2}-2 \mathcal{I}_{M_{a}}\right) g_{\mathrm{GUT}}^{2} \mathcal{I}_{M_{a}}\right)-g^{4} \mathcal{I}_{M_{a}} N^{2}\right\} \\
& +g^{4} \mathcal{I}_{B_{a}}^{2} N\left(b_{a}+N\right)+32 \pi^{2} b_{a} g^{2} g_{\mathrm{GUT}}^{2} \mathcal{I}_{B_{a}} M_{0} N=0, \quad(a=1,2,3),
\end{aligned}
$$

where we have defined

$$
\begin{aligned}
& \mathcal{I}_{B_{a}} \equiv\left(b_{a} m_{3 / 2}-16 \pi^{2} I_{B_{a}}\right) g_{\mathrm{GUT}}^{2} \\
& \mathcal{I}_{M_{a}} \equiv I_{M_{a}}-b_{a} M_{0}^{2} n_{\epsilon_{a}}
\end{aligned}
$$

where

$$
\begin{aligned}
n_{\epsilon_{1}} & \equiv \frac{5}{8}\left(1+n_{D}-n_{e}-n_{U}\right), \\
n_{\epsilon_{2}} & \equiv \frac{1}{24}\left(15-9 n_{D}+n_{e}+9 n_{U}-16 n_{L}\right), \\
n_{\epsilon_{3}} & \equiv \frac{1}{16}\left(5-5 n_{D}+n_{e}-n_{U}\right) .
\end{aligned}
$$

All $m_{3 / 2}$-dependence in $\left(\right.$ III.66 is now contained in $\mathcal{I}_{B_{a}}$, and can be eliminated with the solution ti $m_{3 / 2}(a)$ from (III.13) to obtain

$$
\mathcal{I}_{B_{a}}=16 \pi^{2} g_{\mathrm{GUT}}^{2} \frac{\sum_{b, c} \epsilon_{a b c}\left(b_{a} I_{B_{c}}-b_{c} I_{B_{a}}\right)}{\sum_{d, e} \epsilon_{a d e} b_{e}} .
$$

A solution for $N$ can be obtained from each of the equations (III.66),

$$
\begin{aligned}
N_{(a)} & =-\frac{b_{a}}{2 g^{2}\left(\mathcal{I}_{B_{a}}^{2}-256 \pi^{4} \mathcal{I}_{M_{a}}\right)}\left\{g^{2}\left(\mathcal{I}_{B_{a}}^{2}+256 \pi^{4}\left(-2 \mathcal{I}_{M_{a}}-M_{0}^{2}\right)\right)+32 g_{\mathrm{GUT}}^{2}\left(\pi^{2} \mathcal{I}_{B_{a}} M_{0}+16 \pi^{4} \mathcal{I}_{M_{a}}\right)\right. \\
& \left. \pm\left(\mathcal{I}_{B_{a}}+16 \pi^{2} M_{0}\right) \sqrt{g^{4}\left(\mathcal{I}_{B_{a}}-16 \pi^{2} M_{0}\right)^{2}+64 \pi^{2} g^{2} g_{\mathrm{GUT}}^{2}\left(\mathcal{I}_{B_{a}} M_{0}-16 \pi^{2} \mathcal{I}_{M_{a}}\right)+1024 \pi^{4} g_{\mathrm{GUT}}^{4} \mathcal{I}_{M_{a}}}\right\}
\end{aligned}
$$

Solutions can also be obtained for $M_{0}, n_{\epsilon_{a}}, m_{3 / 2}$, or $g$ :

$$
\begin{aligned}
M_{0(a)}= & \frac{N g^{2}+b_{a} g^{\prime 2}}{n_{\epsilon_{a}}\left(N g^{2}+b g^{\prime 2}\right)^{2}+b g^{\prime 4}+N g^{4}}\left\{ \pm \sqrt{\left(n_{\epsilon_{a}}\left(N g^{2}+b g^{2}\right)^{2}+b g^{4}+N g^{4}\right) I_{M_{a}}-b_{a} g^{4} N\left(n_{\epsilon_{a}} b_{a}^{\prime}+1\right) \mathcal{I}_{B_{a}}^{2}}\right. \\
& \left.-\frac{N b_{a} g^{2}\left(g^{2}-g^{\prime 2}\right) \mathcal{I}_{B_{a}}}{16 \pi^{2}\left(N g^{2}+b_{a} g^{\prime 2}\right)},\right\} \\
n_{\epsilon_{a}}= & \frac{I_{M_{a}}}{b_{a} M_{0}^{2}}-\frac{\left(N g^{2} \mathcal{I}_{B_{a}}-16 \pi^{2} b_{a} g^{\prime 2} M_{0}\right)^{2}+b_{a} N g^{4}\left(\mathcal{I}_{B_{a}}+16 \pi^{2} M_{0}\right)^{2}}{256 \pi^{4} b_{a} M_{0}^{2}\left(N g^{2}+b_{a} g^{\prime 2}\right)^{2}}
\end{aligned}
$$




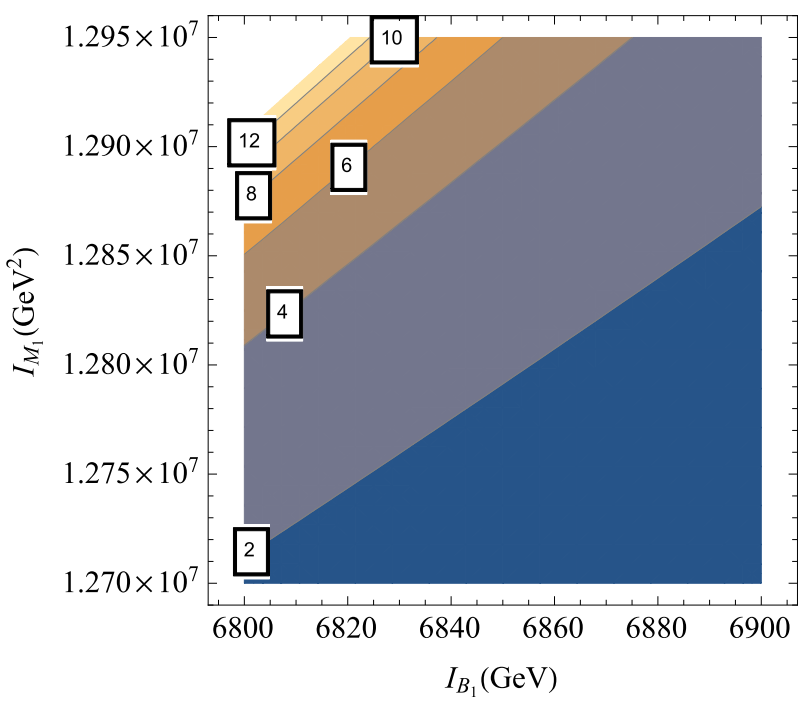

(a) N

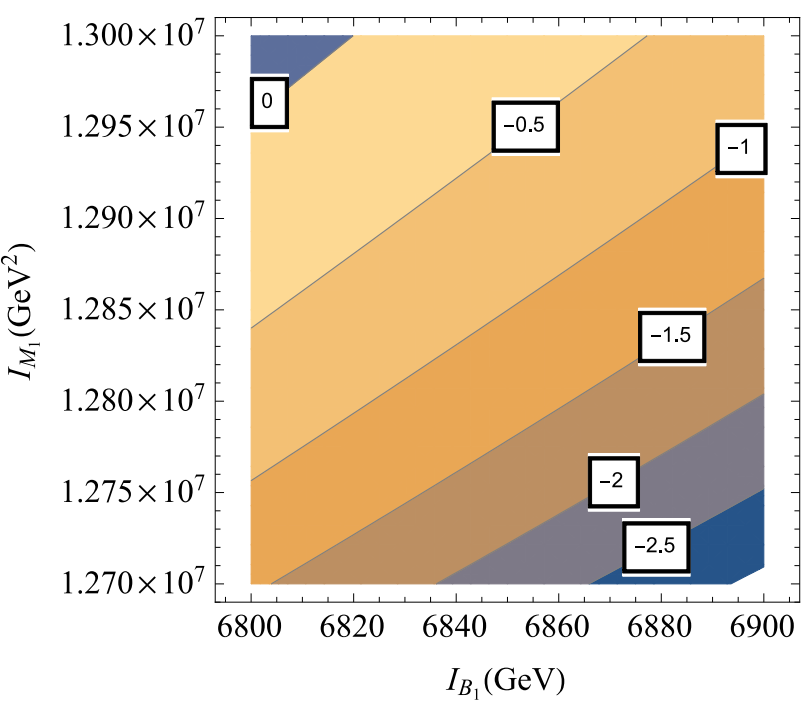

(b) $\alpha_{g}$

FIG. 5: The number of messenger field pairs N, and $\alpha_{g}$ as predicted by III.73 and III.14. $M_{0}=2.0 \mathrm{TeV}, \alpha_{m}=1$, and $g=-\frac{\mathcal{Y}\left(n_{\delta \gamma}\right)}{10 D_{Y \alpha}}=0.80$, and $n_{u}=1 / 2$.

$$
\begin{aligned}
m_{3 / 2(a)} & =16 \pi^{2}\left(\frac{I_{B_{a}}}{b_{a}}-\frac{M_{0}}{g^{2} b_{a}^{\prime}} \pm \frac{\left(N g^{2}+b_{a} g^{2}\right) \sqrt{N\left(b_{a}^{\prime} \mathcal{I}_{M_{a}}-b_{a} M_{0}^{2}\right)}}{N b_{a}^{\prime} b_{a} g^{2}\left(g^{2}-g^{\prime 2}\right)}\right) \\
g_{(a)}^{2} & = \pm \frac{16 \pi^{2} g_{\mathrm{GUT}}^{2} b_{a}\left(\mathcal{I}_{M_{a}}-M_{0}^{2}\right)}{16 \pi^{2}\left(b_{a}^{\prime} \mathcal{I}_{M_{a}}-b_{a} M_{0}^{2}\right)+\mathcal{I}_{B_{a}} M_{0} N \pm\left(\mathcal{I}_{B_{a}}+16 \pi^{2} M_{0}\right) \sqrt{N\left(b_{a}^{\prime} \mathcal{I}_{M_{a}}-b_{a} M_{0}^{2}\right)}},
\end{aligned}
$$

where

$$
\begin{aligned}
b_{a}^{\prime} & =b_{a}+N, \\
g^{\prime 2} & =g^{2}-g_{\mathrm{GUT}}^{2} .
\end{aligned}
$$

The correct signs have to be determined by other means, such as by comparing solutions and ruling out negative values. Only the solutions with a different index are independent; i.e. we cannot use the solution $N=N_{(1)}$ to solve $M_{0(1)}$ etc. Three linearly independent solutions can be "picked", each with a different index, e.g. $M_{0(1)}$, $N_{(2)}, g_{(3)}$, and the supplemented with the solutions obtained from $D_{I}$ and $I_{B_{a}}$.

Fig. 5. illustrates the behavior of $\mathrm{N}$ and $\alpha_{g}$ as a function of $I_{B_{1}}$ and $I_{M_{1}}$ as predicted by (III.73) and (III.14). We assume $n_{u}=1 / 2$, and $D_{Y \alpha}, D_{Y_{13 H}}, D_{\chi_{1}}$, and two of the $I_{B_{a}}$ to have been measured to fix $g=0.80, \alpha_{m}=1$, and $M_{0}=2 \mathrm{TeV}$. Thus $I_{B_{1}}$ and $I_{M_{1}}$ determine the values of $N$ and $\alpha_{g}$. Small variation in $I_{M_{1}}$ and $I_{B_{1}}$ point to significantly different values of $N$ and $\alpha_{g}$; if $I_{M_{1}}=1.29 \times 10^{7} \mathrm{GeV}^{2}$, a difference of $50 \mathrm{GeV}$ in $I_{B_{1}}$ separates $N=4$ and $N=12$. Table III, summarizes the solutions derived above.

\section{The modular weights.}

In terms of unimodular weights $n_{h}=n_{H_{d}}=n_{H_{u}}$ and $n_{u}=n_{U}=n_{D}=n_{L}=n_{E}=n_{Q}$, for which

$$
\begin{aligned}
n_{\alpha} & =n_{h}-n_{u}, \\
n_{\beta} & =5\left(1-n_{u}\right), \\
n_{\gamma} & =n_{\delta}=n_{\delta \gamma}=0, \\
n_{\epsilon_{1}} & =\frac{5}{8}\left(1-n_{u}\right), \\
n_{\epsilon_{2}} & =\frac{5}{8}\left(1-n_{u}\right), \\
n_{\epsilon_{3}} & =\frac{5}{16}\left(1-n_{u}\right),
\end{aligned}
$$




\begin{tabular}{|c|c|c|c|}
\hline Condition & $I_{B_{a}}(T e V)=I_{B_{a}}(G U T)+\Delta I_{B_{a}}$ & $D_{I}(T e V)=D_{I}(G U T)$ & $\begin{array}{c}I_{M_{a}}(T e V)=I_{M_{a}}(G U T)+\Delta I_{M_{a}} \\
I I I .14\end{array}$ \\
\hline Solution & $\begin{array}{ll}3 \times\left\{m_{3 / 2}\right. & I I I .13 \\
3 \times\left\{\alpha_{g}\left(M_{0}, N, m_{3 / 2}\right)\right. & I I I .14\end{array}$ & 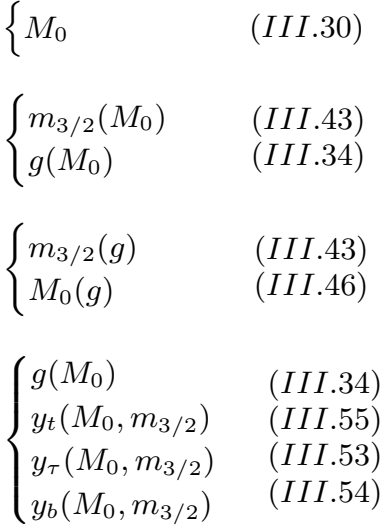 & $\begin{array}{l}3 \times\left\{N\left(M_{0}, m_{3 / 2}, g\right)\right. \\
3 \times\left\{M_{0}\left(N, m_{3 / 2}, g\right)\right. \\
3 \times\left\{g\left(N, M_{0}, m_{3 / 2},\right)\right. \\
3 \times\left\{m_{3 / 2}\left(N, M_{0}, g,\right)\right.\end{array}$ \\
\hline
\end{tabular}

TABLE III: Summary of the solutions for the parameters derived in Chapter III.

the $M_{0}$-term is removed from the solution of $g$, thus III.34 is simplified to

$$
g^{2}=-\frac{13 D_{Y_{13 H}}}{10 D_{Y \alpha}}
$$

Determining an analytical solution for $n_{u}$ requires solving two of the three equations (III.66) simultaneously for $N$ and $n_{u}$. While the solution exists, it is too complex to be presented explicitly. A simpler way may be to determine $n_{u}$ numerically or by guessing from the equation

$$
N_{(1)}=N_{(2)}
$$

The third independent equation $N_{(3)}$ then remains for determining $N . M_{0}, m_{3 / 2}, \mu_{\mathrm{MESS}}$, and $N$ are now known, thus the Yukawas at the GUT-scale can be evaluated through conventional method of the renormalization group running. Then $n_{h}$ can be determined from

$$
D_{Z}=2 M_{0}^{2}\left(n_{h}-n_{u}\right)+\left(Y_{Z a}+Y_{Z b} g^{2}\right) m_{3 / 2}^{2} .
$$

A measurement of $D_{\chi_{1}}=0$ would present the problem of determining whether $M_{0}=0$ or $n_{u}=1$. If we assume the latter, two of the equations (III.66) could be solved simultaneously for $M_{0}$ and $N$. Then the value of $m_{3 / 2}$ from the remaining independent equation (III.76) can be evaluated and checked for consistency with (III.13), to verify the hypothesis.

In the general case it is not possible to determine all seven combinations of the modular weights since after solving the five parameters only five independent RGIs remain. left. Some of $n_{\epsilon_{a}}$ can be solved from (III.75) and sum rules discussed in Section IV could be helpful in determining $n_{\beta}, n_{\alpha}, n_{\gamma}$, and $n_{\delta}$.

\section{SUM RULES IN DEFLECTED MIRAGE MEDIATION}

Renormalization group invariants can be used to construct sum rules by applying various conditions to reduce variables, e.g. mass unification. As a generic example we assume gaugino mass unification at some scale and write $M_{1}=M_{2}=M_{3}=M_{1 / 2}$. From (III.3),

$$
I_{B_{a}}=\frac{M_{1 / 2}}{g_{a}^{2}}
$$

By combining this to the definitions of the invariants $I_{g_{2}}$ and $I_{g_{3}}$,

$$
\begin{aligned}
& I_{g_{2}}=1 / g_{1}^{2}-\left(b_{1} / b_{2}\right) g_{2}^{-2}, \\
& I_{g_{3}}=1 / g_{1}^{2}-\left(b_{1} / b_{3}\right) g_{3}^{-2},
\end{aligned}
$$




\begin{tabular}{|c|c|c|}
\hline Sum rule & $\mu<\mu_{\text {mess }}$ & $\mu>\mu_{\text {mess }}$ \\
\hline$I_{g_{1}}-I_{g_{2}}\left(1-b_{1} / b_{2}\right) /\left(1-b_{1} / b_{3}\right)=0$ & OK & OK \\
\hline$\left(I_{B_{1}}-\left(b_{1} / b_{3}\right) I_{B_{3}}\right) I_{g_{2}}=\left(I_{B_{1}}-\left(b_{1} / b_{2}\right) I_{B_{2}}\right) I_{g_{3}}$ & OK & OK \\
\hline$I_{g_{2}}=\left(I_{M_{1}}-\frac{b_{1}}{8} D_{\chi_{1}}\right)^{-1 / 2} I_{B_{1}}-\frac{b_{1}}{b_{2}}\left(I_{M_{2}}-\frac{b_{2}}{8} D_{\chi_{1}}\right)^{-1 / 2} I_{B_{2}}$ & $\mathrm{X}$ & $n_{i}=n_{u}$ \\
\hline$I_{g_{3}}=\left(I_{M_{1}}-\frac{b_{1}}{8} D_{\chi_{1}}\right)^{-1 / 2} I_{B_{1}}+\frac{b_{1}}{b_{3}}\left(I_{M_{3}}+\frac{b_{3}}{16} D_{\chi_{1}}\right)^{-1 / 2} I_{B_{3}}$ & $\mathrm{X}$ & $n_{i}=n_{u}$ \\
\hline$I_{M_{1}}-\left(2 b_{1}+b_{3}\right) /\left(2 b_{2}+b_{3}\right) I_{M_{2}}+2\left(b_{1}-b_{2}\right) /\left(2 b_{2}+b_{3}\right) I_{M_{3}}=0$ & $\mathrm{X}$ & $n_{i}=1$ \\
\hline
\end{tabular}

TABLE IV: Sum rules derived from the condition of gauge coupling unification, gaugino mass unification and scalar mass unification. Third and fourth rows describe whether the sum rule is valid above and below messenger scale respectively. The bottom three sum rules involving scalar masses are valid only above messenger scale and with the condition of unimodular weights $n_{Q}=n_{U}=n_{D}=n_{E}=n_{L}=n_{u}$. Above the messenger scale $b_{a}^{\prime}$ is to be substituted for $b_{a}$.

we can eliminate $M_{1 / 2}$ from the resulting group of equations to obtain the sum rule

$$
\left[I_{B_{1}}-\left(b_{1} / b_{3}\right) I_{B_{3}}\right] I_{g_{2}}=\left[I_{B_{1}}-\left(b_{1} / b_{2}\right) I_{B_{2}}\right] I_{g_{3}}
$$

If we assume the gaugino mass unification to occur in conjunction with a scalar mass unification (with the common scalar mass squared value $m_{0}^{2}$ ) at the same scale, the RGIs $I_{M_{a}}$ have the values

$$
\begin{aligned}
& I_{M_{1}}=\frac{33 m_{0}^{2}}{8}+M_{1 / 2}^{2}, \\
& I_{M_{2}}=\frac{5 m_{0}^{2}}{8}+M_{1 / 2}^{2}, \\
& I_{M_{3}}=M_{1 / 2}^{2}-\frac{15 m_{0}^{2}}{16} .
\end{aligned}
$$

This allows us to write the sum rule

$$
81 I_{M_{2}}-56 I_{M_{3}}-25 I_{M_{1}}=0 \text {. }
$$

Similarly from the assumption of gauge coupling unification at the GUT scale one can derive

$$
I_{g_{1}}-I_{g_{2}}\left(1-b_{1} / b_{2}\right) /\left(1-b_{1} / b_{3}\right)=0
$$

In the specific case of deflected mirage mediation we can verify the formula easily for renormalization scales above the messenger scale by substituting $b_{a}^{\prime}$ for $b_{a}$ and plugging in the GUT-scale values for the gaugino masses (II.4). To examine the equation below the messenger scale we restore $b_{a}$ and write the invariants at the scale $\mu_{\text {mess }}^{-}$Using (IV.3), (III.7), and (III.5) it is straightforward to verify that (IV.4) is valid below messenger scale as well. Similarly one can derive sum rules based on scalar mass unification and scalar mass unification combined to gaugino mass unification at the same unification scale [37. Validity of the sum rules derived in 37] at regions above and below the messenger scale is listed in Table IV for deflected mirage mediation.

We will attempt to derive sum rules valid at all energies using the RGIs, that are not dependent on other parameters than $n_{i}$ and $N$. From Table II we see that $D_{L_{13}}=0$ and thus we immediately have

$$
2\left(m_{\tilde{L}_{1}}^{2}-m_{\tilde{L}_{3}}^{2}\right)-m_{\tilde{e}_{1}}^{2}+m_{\tilde{e}_{3}}^{2}=0 .
$$

More sum rules can be constructed by combining the solutions to the mass parameters and $g$. For instance 
by equating the two expressions for $m_{3 / 2}$; (III.13), and (III.76) with $a=1$, we obtain

$$
\begin{aligned}
\frac{165}{48} g^{4} g_{\mathrm{GUT}}^{4} N(5 N+33)\left(I_{B_{1}}-I_{B_{3}}\right)= & \sqrt{5} g^{2} g_{\mathrm{GUT}}^{2}\left(g^{2}(5 N+33)-33 g_{\mathrm{GUT}}^{2}\right) \sqrt{N\left(\mathcal{I}_{M_{1}} n_{\beta}(5 N+33)-\frac{33}{n_{\beta}} D_{\chi_{1}}\right)} \\
& +5 g^{4} g_{\mathrm{GUT}}^{4} I_{B_{1}} N(5 N+33)-165 g^{2} g_{\mathrm{GUT}}^{4} N \sqrt{\frac{D_{\chi_{1}}}{n_{\beta}}}, \quad \text { (IV.11) }
\end{aligned}
$$

where we have again used $M_{0}^{2}=\frac{D_{\chi_{1}}}{n_{\beta}}$ and $g^{2}=\frac{-\mathcal{Y}\left(n_{\delta \gamma}\right)}{10 D_{Y \alpha}}$. Similarly from the solutions of $g^{2}$;III.39) and III.77), we obtain

$$
\begin{aligned}
& -10 n_{\delta \gamma} D_{\chi_{1}}-13 D_{Y_{13 H}} n_{\beta} \\
& =\frac{160 \pi^{2} g_{\mathrm{GUT}}^{2} D_{Y \alpha}\left(n_{\beta} \mathcal{I}_{M_{2}}-D_{\chi_{1}}\right)}{\left.16 \pi^{2}\left((1+N) \mathcal{I}_{M_{2}}-\frac{a D_{\chi_{1}}}{n_{\beta}}\right)+\mathcal{I}_{B_{2}} \sqrt{\frac{D_{\chi_{1}}}{n_{\beta}}} N-\left(\mathcal{I}_{B_{2}}+16 \pi^{2} \sqrt{\frac{D_{\chi_{1}}}{n_{\beta}}}\right) \sqrt{N\left((1+N) \mathcal{I}_{M_{a}}-\frac{D_{\chi_{1}}}{n_{\beta}}\right.}\right)} .
\end{aligned}
$$

To construct sum rules independent on $N$, one can combine to solutions III.73 to form three sum rules

$$
N_{(1)}=N_{(2)}=N_{(3)} \text {. }
$$

We leave out the explicit formula for brevity. This provides a way to determine some of the modular weights. Additional sum rules can be formed in a similar manner from (III.43, (III.45), (III.46), and (III.74) or by combining other solutions summarized in Table III.

\section{A. Special cases}

In order to draw distinctions between the supersymmetry breaking scenarios and identify dominating contributions we consider sum rules in some special cases obtained from the deflected mirage mediation boundary conditions.

\section{The case $N=0$}

In the absence of messenger fields, $\Delta I_{B_{a}}=\Delta I_{M_{a}}=0$, and all the invariants have equal values at the TeV-scale and the GUT-scale. As noted before, since the threshold correction for $I_{B_{a}}$,

$$
\Delta I_{B_{a}}=-N M_{0} /\left(16 \pi^{2}\right)\left(1+\alpha_{g}\right) \alpha_{m} \ln \frac{M_{P}}{m_{3 / 2}},
$$

vanishes for $\alpha_{g}=-1$ as well as for $N=0$, we cannot deduce the absence of the messenger fields from the condition $I_{B_{a}}\left(\mu_{\mathrm{TEV}}\right)=I_{B_{a}}\left(\mu_{\mathrm{GUT}}\right)$ alone. On the other hand, from (III.11), the threshold correction for $I_{M_{1}}$ reads

$$
\begin{aligned}
\Delta I_{M_{1}}= & -2 M_{1} \Delta M_{1}-\Delta M_{1}^{2}+\frac{5 N}{8}\left(m_{\tilde{d}_{1}}^{2}\left(\mu_{\mathrm{MESS}}^{+}\right)-m_{\tilde{u}_{1}}^{2}\left(\mu_{\mathrm{MESS}}^{+}\right)-m_{\tilde{e}_{1}}^{2}\left(\mu_{\mathrm{MESS}}^{+}\right)\right) \\
& -\frac{5 b_{1}}{8}\left(\Delta m_{\tilde{d}_{1}}^{2}-\Delta m_{\tilde{u}_{1}}^{2}-\Delta m_{\tilde{e}_{1}}^{2}\right) .
\end{aligned}
$$

If $\alpha_{g}=-1$, the corrections to scalar and gaugino masses vanish, leaving

$$
\Delta I_{M_{1}}=\frac{5 N}{8}\left(m_{\tilde{d}_{1}}^{2}\left(\mu_{\mathrm{MESS}}^{+}\right)-m_{\tilde{u}_{1}}^{2}\left(\mu_{\mathrm{MESS}}^{+}\right)-m_{\tilde{e}_{1}}^{2}\left(\mu_{\mathrm{MESS}}^{+}\right)\right), \quad \text { for } \alpha_{g}=-1
$$

As one can see, $\Delta I_{M_{1}}=0$ only if $N=0$ (or $m_{\tilde{d}_{1}}^{2}-m_{\tilde{u}_{1}}^{2}-m_{\tilde{e}_{1}}^{2}=0$, which would imply a constant $M_{1}$ above the messenger scale). Similar condition obviously applies for $I_{M_{2}}$ and $I_{M_{3}}$. Consequently, the equation $I_{M_{a}}\left(\mu_{\mathrm{GUT}}\right)=I_{M_{a}}\left(\mu_{\mathrm{TEV}}\right)$ provides us with a better condition for the absence of messengers. Thus we write,

$$
I_{M_{a}}\left(\mu_{\mathrm{TEV}}\right)=I_{M_{a}}\left(\mu_{\mathrm{GUT}}\right)=M_{0}^{2}\left(1+b_{a} n_{\epsilon_{a}}\right), \quad \text { for } N=0 .
$$


From [III.69)-III.71] we see that in the case of universal modular weights $n_{\epsilon_{1}}=n_{\epsilon_{2}}=2 n_{\epsilon_{3}}=5 / 8\left(1-n_{u}\right)$. Now the $n_{\epsilon_{a}}$ can be eliminated from (IV.17) and three conditions obtained,

$$
\begin{aligned}
& M_{0}=\frac{\sqrt{3 I_{M_{2}}+2 I_{M_{3}}}}{\sqrt{5}}, \\
& 5 / 8\left(1-n_{u}\right)=\frac{2\left(I_{M_{2}}-I_{M_{3}}\right)}{3 I_{M_{2}}+2 I_{M_{3}}}, \\
& 81 I_{M_{2}}-56 I_{M_{3}}-25 I_{M_{1}}=0 .
\end{aligned}
$$

The equation (IV.20 can in fact also be derived from the assumptions of scalar mass and gaugino mass unification at the same scale without additional assumptions about the supersymmetry breaking scenario [37. We then use the solution $M_{0}^{2}=\frac{D_{\chi_{1}}}{5\left(1-n_{u}\right)}$ from III.30, and the first two equations yield (after eliminating $n_{u}$ and $\left.M_{0}\right)$,

$$
D_{\chi_{1}}=16 / 5\left(I_{M_{2}}-I_{M_{3}}\right), \quad \text { for } N=0, n_{u} \neq 1 .
$$

The equations (IV.20) and (IV.21) now provide a simple test for determining the existence of the messenger sector, with the caveat that universal modular weights are required.

$$
\text { 2. The cases } M_{0}=0, m_{3 / 2}=0, g=g_{G U T}
$$

In the case of zero $M_{0}$ and $m_{3 / 2}$ we obtain from (III.30, , III.13),

$$
\begin{aligned}
& D_{\chi_{1}}=0, \quad \text { for } M_{0}=0, \\
& \sum_{b, c=1}^{3} \epsilon_{a b c} I_{B_{c}}=0, \quad(a=1,2,3), \quad \text { for } m_{3 / 2}=0 .
\end{aligned}
$$

The case $g=g_{\mathrm{GUT}}$ implies either $\mu_{\mathrm{MESS}}=\mu_{\mathrm{GUT}}$ or $N=0$. From III.34

$$
13 D_{Y_{13 H}}+10 M_{0}^{2}\left(n_{\gamma}+n_{\delta}\right)=10 g_{\mathrm{GUT}}^{2} D_{Y \alpha} .
$$

If we assume universal modular weights this is simplified to

$$
13 D_{Y_{13 H}}=10 g_{\mathrm{GUT}}^{2} D_{Y \alpha} .
$$

\section{COMPARISON WITH SUGRA, AMSB, GAUGE MEDIATION AND PURE MIRAGE MEDIATION}

Deflected mirage mediation includes contributions from three separate supersymmetry breaking mechanisms, namely gravity mediation (SUGRA) 3, 44, 47, gauge mediation (GMSB) 48 50, and anomaly mediation (AMSB) [51, 52]. The boundary conditions for the scalar and the gaugino masses can be parametrized as

$$
\begin{array}{llr}
\text { SUGRA: } & m_{i}^{2}\left(\mu_{\mathrm{GUT}}\right)=\left(1-n_{i}\right) M_{0}^{2} ; & M_{a}^{2}\left(\mu_{\mathrm{GUT}}\right)=M_{0}^{2}, \\
\text { AMSB: } & m_{i}^{2}\left(\mu_{\mathrm{GUT}}\right)=-\frac{\dot{\gamma}_{i}^{\prime}}{\left(16 \pi^{2}\right)^{2}} m_{3 / 2}^{2} ; & M_{a}^{2}\left(\mu_{\mathrm{GUT}}\right)=g^{2} \frac{b_{a}}{16 \pi^{2}} m_{3 / 2}, \\
\text { GMSB: } & m_{i}^{2}\left(\mu_{\mathrm{MESS}}\right)=\frac{N \Lambda^{2}}{\left(16 \pi^{2}\right)^{2}} \sum_{a=1}^{3} c_{a}\left(\Psi_{i}\right) g_{a}^{4} ; & M_{a}^{2}\left(\mu_{\mathrm{MESS}}\right)=-\frac{N g_{a}^{2}}{16 \pi^{2}} \Lambda .
\end{array}
$$

Note that the GMSB boundary conditions are defined on the messenger scale possibly different from the GUT scale while the AMSB and SUGRA boundary conditions are defined at the GUT-scale. Additionally, two combinations of the above exist: mirage mediation (MMSB) [6] is obtained from DMMSB by removing the messengers and deflected anomaly mediation (DAMSB) 53 55 is obtained by setting $M_{0}$ to zero.

The boundary conditions for the five models can be extracted from (II.4), (II.5), (II.11), and (II.10) with the following prescriprions: 


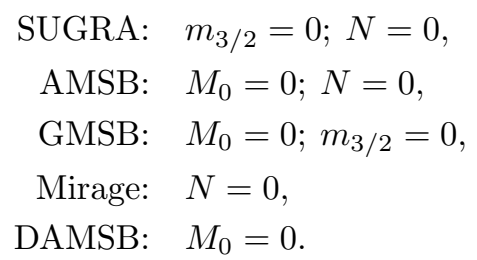

Here we assume universal modular weights for all applicable models and the parameters not specified to be nonzero. In the case that one of the mechanisms clearly dominates supersymmetry breaking scenario can then in principle be resolved or narrowed by measuring the parameters in terms of RGIs. By comparing to the solutions for the parameters in terms of invariants (III.13), (III.30), and (III.41), along with the sum rules IV.20- IV.25, we can deduce from the measured invariants $I_{B_{1}}, I_{B_{3}}, I_{M_{a}}, \overline{D_{Y \alpha}}$, and $D_{Y_{13 H}}$ the following:

$$
\begin{gathered}
I_{B_{1}}-I_{B_{3}} \propto m_{3 / 2}\left\{\begin{array}{l}
0, \text { for mSUGRA, GMSB } \\
>0, \text { for AMSB,Mirage, DAMSB }
\end{array}\right. \\
D_{\chi_{1}}=\left(1-n_{u}\right) M_{0}^{2}\left\{\begin{array}{l}
=0, \text { for AMSB, GMSB } \\
\propto\left(1-n_{u}\right), \text { for mSUGRA, Pure Mirage, DAMSB }
\end{array}\right. \\
\frac{10 D_{Y \alpha}}{13 D_{Y_{13 H}}}+\frac{1}{g_{\mathrm{GUT}}^{2}}=\frac{1}{g_{\mathrm{GUT}}^{2}}-\frac{1}{g^{2}}\left\{\begin{array}{l}
=0 \text { for mSUGRA, AMSB, Pure Mirage } \\
\propto t_{\mathrm{MESS}} N, \text { for GMSB, DAMSB }
\end{array}\right. \\
81 I_{M_{2}}-56 I_{M_{3}}-25 I_{M_{1}} \begin{cases}=0, & \text { for AMSB, mSUGRA, Pure Mirage } \\
\neq 0, & \text { for Deflected Mirage, GMSB, DAMSB }\end{cases}
\end{gathered}
$$

Thus e.g. observing $D_{\chi_{1}}=0$ would exclude mediation mechanism with a gravity mediated contribution with $n_{u} \neq 1$, but deflected or pure mirage with $n_{u}=1$ cannot be ruled out. On the other hand a nonzero $\frac{10 D_{Y \alpha}}{13 D_{Y_{13 H}}}+\frac{1}{g_{\mathrm{GUT}}^{2}}$ implicates a gauge mediated contribution, with the messenger scale different from the GUT scale. We have illustrated the implications of different values of $(\mathrm{V} .12)$ in Fig. 6 by starting from the measurement of $D_{\chi_{1}}$.

\section{CONCLUSIONS}

Deflected mirage mediation is the most general type of mechanism for spontaneous supersymmetry breaking in the sense that it includes contributions from three SSB mechanisms, namely gravity-, anomaly-, and gauge mediation. The renormalization group invariants provide a way of determining the values of the supersymmetry breaking parameters, but in the case of DMMSB, the emergence of gauge messenger fields at a scale possibly different from the GUT-scale complicates their use by inducing corrections to the gaugino and the scalar masses and modifying the beta functions at this threshold. Thus the invariants have differing values above and below the messenger scale. In order to connect the TeV scale measurements of the particle masses to the GUTscale parameters we have derived the threshold corrections to the RGIs and derived the RGIs for arbitrary $b_{a}$-coefficients of the beta functions. It is shown that the high scale parameters which include $N, \mu_{\mathrm{MESS}}, m_{3 / 2}$, $M_{0}$, and $\alpha_{g}$ can be analytically solved in terms of the RGIs, and the explicit formulas are provided. We have then examined various limits of DMMSB to see how any of the contributing three pure supersymmery breaking scenarios can be detected by measuring the RGIs at the TeV scale.

We have also discussed how the solutions to the supersymmetry breaking parameters can be used to construct sum rules that would allow further testing of the theory and determine the modular weights for the scalar masses. The lightest Higgs mass measurement allows the restriction of the parameter space of DMMSB. We have discussed the implications of the measured Higgs mass of $125.7 \mathrm{GeV}$ to the mass spectrum and the parameters of DMMSB.

\section{ACKNOWLEDGMENTS}

KH and PT acknowledge support from the Academy of Finland (Project No 137960). PNP would like to thank Department of Physics, University of Helsinki, and Helsinki Institute of Physics for hospitality while 


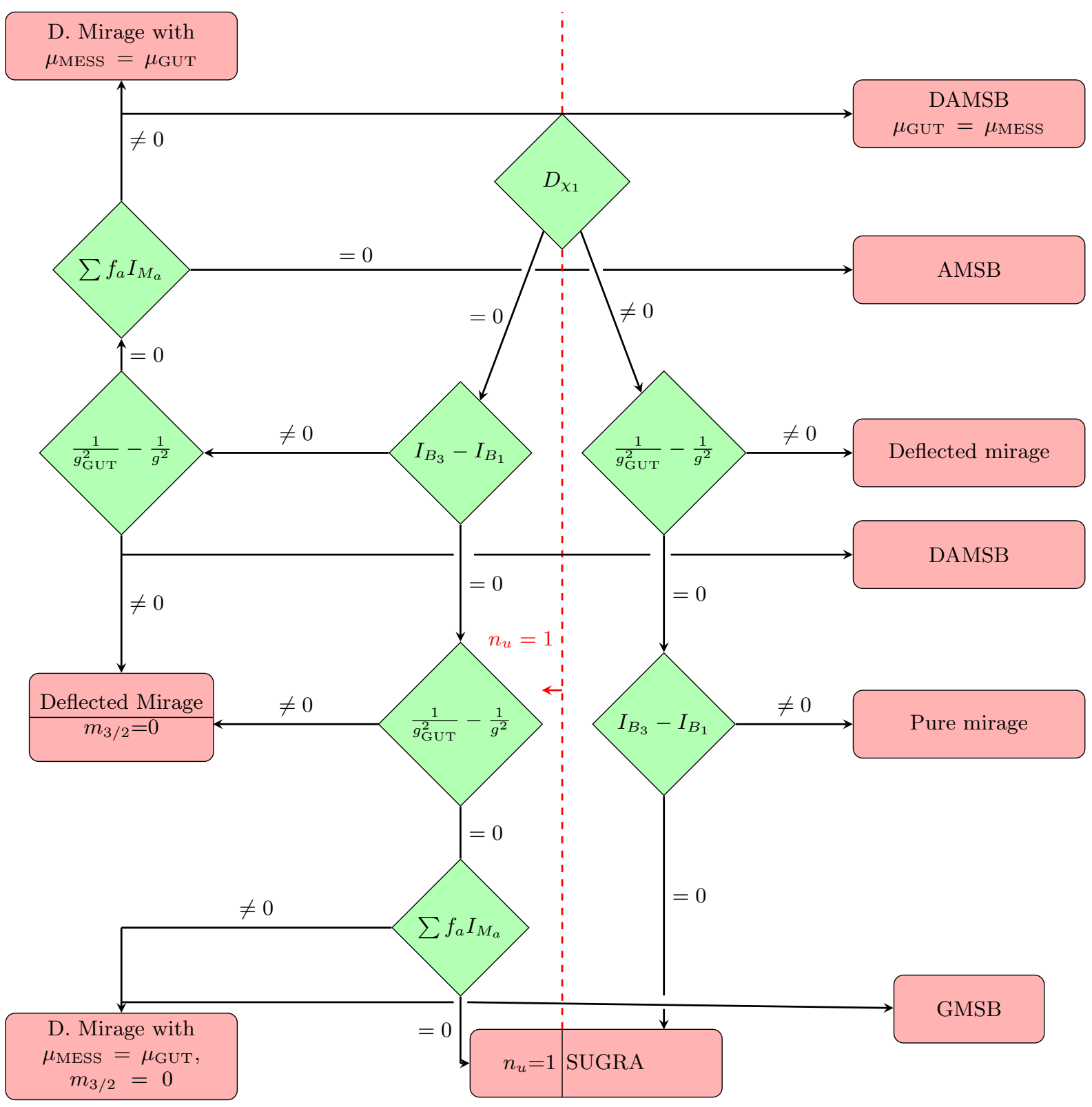

FIG. 6: Implications of measurement of RGIs for the supersymmetry breaking mechanism with the assumption of $n_{h}=n_{H_{d}}=n_{H_{u}}$ and $n_{u}=n_{U}=n_{D}=n_{L}=n_{E}=n_{Q}$, assuming that one or two of the mechanisms dominate. On the left side of the red dotted line all endpoints have $n_{u}=1 . \frac{1}{g^{2}}=-\frac{10 D_{Y \alpha}}{13 D_{Y_{13 H}}}$ and $\sum f_{a} I_{M_{a}}=81 I_{M_{2}}-56 I_{M_{3}}-25 I_{M_{1}}$.

part of this work was done. The work of PNP is supported by Raja Ramanna Fellowship of the Department of Atomic Energy, and partly by J. C. Bose National Fellowship of the Department of Science and Technology, India.

[1] ATLAS Collaboration, https://twiki.cern.ch/twiki/bin/view/AtlasPublic/SupersymmetryPublicResults.

[2] CMS Collaboration, https://twiki.cern.ch/twiki/bin/view/CMSPublic/PhysicsResultsSUS.

[3] A. H. Chamseddine, R. L. Arnowitt and P. Nath, Phys. Rev. Lett. 49, 970 (1982).

[4] M. Dine, A. E. Nelson and Y. Shirman, Phys. Rev. D 51, 1362 (1995) hep-ph/9408384.

[5] L. Randall and R. Sundrum, Nucl. Phys. B 557, 79 (1999) hep-th/9810155.

[6] S. Kachru, R. Kallosh, A. D. Linde and S. P. Trivedi, Phys. Rev. D 68, 046005 (2003) hep-th/0301240.

[7] K. Choi, A. Falkowski, H. P. Nilles, M. Olechowski and S. Pokorski, JHEP 0411, $076(2004)$ hep-th/0411066. 
[8] K. Choi, K. S. Jeong, T. Kobayashi and K. i. Okumura, Phys. Lett. B 633, 355 (2006) hep-ph/0508029.

[9] K. Choi, K. S. Jeong and K. i. Okumura, JHEP 0509, 039 (2005) hep-ph/0504037.

[10] K. Choi and H. P. Nilles, JHEP 0704, 006 (2007) hep-ph/0702146 [HEP-PH]].

[11] K. Choi, K. S. Jeong and K. i. Okumura, JHEP 0509, 039 (2005) hep-ph/0504037.

[12] L. L. Everett, I. W. Kim, P. Ouyang and K. M. Zurek, JHEP 0808, 102 (2008) arXiv:0806.2330 [hep-ph]].

[13] U. Chattopadhyay, A. Corsetti and P. Nath, Phys. Rev. D 66, 035003 (2002) hep-ph/0201001.

[14] U. Chattopadhyay, A. Corsetti and P. Nath, Phys. Rev. D 68, 035005 (2003) hep-ph/0303201.

[15] A. Djouadi, M. Drees and J. L. Kneur, JHEP 0603, 033 (2006) hep-ph/0602001.

[16] E. A. Baltz, M. Battaglia, M. E. Peskin and T. Wizansky, Phys. Rev. D 74, 103521 (2006) hep-ph/0602187.

[17] B. C. Allanach, K. Cranmer, C. G. Lester and A. M. Weber, JHEP 0708, 023 (2007) arXiv:0705.0487][hep-ph]].

[18] C. F. Berger, J. S. Gainer, J. L. Hewett and T. G. Rizzo, JHEP 0902, 023 (2009) arXiv:0812.0980 [hep-ph]].

[19] R. Lafaye, T. Plehn and D. Zerwas, hep-ph/0404282

[20] M. Carena, P. H. Chankowski, M. Olechowski, S. Pokorski and C. E. M. Wagner, Nucl. Phys. B 491, 103 (1997) hep-ph/9612261.

[21] J. L. Kneur and G. Moultaka, Phys. Rev. D 59, 015005 (1999) hep-ph/9807336].

[22] G. A. Blair, W. Porod and P. M. Zerwas, Eur. Phys. J. C 27, 263 (2003) hep-ph/0210058.

[23] G. A. Blair, A. Freitas, H.-U. Martyn, G. Polesello, W. Porod and P. M. Zerwas, Acta Phys. Polon. B 36, 3445 (2005) hep-ph/0512084.

[24] K. Huitu, J. Laamanen and P. N. Pandita, Phys. Rev. D 65, 115003 (2002) hep-ph/0203186.

[25] B. Ananthanarayan and P. N. Pandita, Mod. Phys. Lett. A 19, 467 (2004) hep-ph/0312361.

[26] B. Ananthanarayan and P. N. Pandita, Int. J. Mod. Phys. A 20, 4241 (2005) hep-ph/0412125].

$[27]$ K. Huitu, J. Laamanen, P. N. Pandita and S. Roy, Phys. Rev. D 72, 055013 (2005) hep-ph/0502100.

[28] B. Ananthanarayan and P. N. Pandita, Int. J. Mod. Phys. A 22, 3229 (2007) arXiv:0706.2560 [hep-ph]].

[29] K. Huitu, J. Laamanen, P. N. Pandita and P. Tiitola, Phys. Rev. D 82, 115003 (2010) arXiv:1006.0661 [hep-ph]].

[30] K. Huitu, P. N. Pandita and P. Tiitola, Phys. Lett. B 716, 298 (2012) arXiv:1103.4782 [hep-ph]].

[31] D. J. Miller, A. P. Morais and P. N. Pandita, Phys. Rev. D 87, no. 1, 015007 (2013) arXiv:1208.5906 [hep-ph]].

[32] D. A. Demir, JHEP 0511, 003 (2005) hep-ph/0408043.

[33] P. Meade, N. Seiberg and D. Shih, Prog. Theor. Phys. Suppl. 177, 143 (2009) [arXiv:0801.3278 [hep-ph]].

[34] M. Carena, P. Draper, N. R. Shah and C. E. M. Wagner, Phys. Rev. D 82, 075005 (2010) arXiv:1006.4363 [hep-ph]].

[35] M. Carena, P. Draper, N. R. Shah and C. E. M. Wagner, Phys. Rev. D 83, 035014 (2011) arXiv:1011.4958 [hep-ph]].

[36] M. Carena, J. Lykken, S. Sekmen, N. R. Shah and C. E. M. Wagner, Phys. Rev. D 86, 075025 (2012) arXiv:1205.5903 [hep-ph]].

[37] J. Hetzel and W. Beenakker, JHEP 1210, 176 (2012) arXiv:1204.4336 [hep-ph]].

[38] J. Hetzel, arXiv:1211.1157 [hep-ph].

[39] S. P. Martin and P. Ramond, Phys. Rev. D 48 (1993) 5365 hep-ph/9306314.

[40] L. L. Everett, I. W. Kim, P. Ouyang and K. M. Zurek, Phys. Rev. Lett. 101, 101803 (2008) arXiv:0804.0592 [hep-ph]].

[41] H. Abe and J. Kawamura, JHEP 1407, 077 (2014) arXiv:1405.0779 [hep-ph]].

[42] M. Carena, J. R. Espinosa, M. Quiros and C. E. M. Wagner, Phys. Lett. B 355, 209 (1995) hep-ph/9504316.

[43] K. A. Olive et al. [Particle Data Group Collaboration], Chin. Phys. C 38, 090001 (2014).

[44] R. Barbieri, S. Ferrara and C. A. Savoy, Phys. Lett. B 119, 343 (1982).

[45] L. E. Ibanez, Phys. Lett. B 118, 73 (1982).

[46] L. J. Hall, J. D. Lykken and S. Weinberg, Phys. Rev. D 27, 2359 (1983).

[47] N. Ohta, Prog. Theor. Phys. 70, 542 (1983).

[48] M. Dine and A. E. Nelson, Phys. Rev. D 48, 1277 (1993) hep-ph/9303230.

[49] M. Dine, A. E. Nelson and Y. Shirman, Phys. Rev. D 51, 1362 (1995) hep-ph/9408384.

[50] M. Dine, A. E. Nelson, Y. Nir and Y. Shirman, Phys. Rev. D 53, 2658 (1996) hep-ph/9507378.

[51] M. Cavaglia, L. Fatibene and M. Francaviglia, Class. Quant. Grav. 15, 3627 (1998) hep-th/9801155.

[52] G. F. Giudice, M. A. Luty, H. Murayama and R. Rattazzi, JHEP 9812, 027 (1998) hep-ph/9810442

[53] A. Pomarol and R. Rattazzi, JHEP 9905 (1999) 013 hep-ph/9903448.

[54] N. Okada, Phys. Rev. D 65 (2002) 115009 hep-ph/0202219.

[55] F. Wang, W. Wang, J. M. Yang and Y. Zhang, arXiv:1505.02785 [hep-ph]. 